\title{
Steepest-Descent Approach to Triple Hierarchical Constrained Optimization Problems
}

\author{
Lu-Chuan Ceng, ${ }^{1}$ Cheng-Wen Liao, ${ }^{2}$ Chin-Tzong Pang, ${ }^{3}$ and Ching-Feng Wen ${ }^{4}$ \\ ${ }^{1}$ Department of Mathematics, Shanghai Normal University, and Scientific Computing Key Laboratory of Shanghai Universities, \\ Shanghai 200234, China \\ ${ }^{2}$ Department of Information Management, Yuan Ze University, Chung-Li 32003, Taiwan \\ ${ }^{3}$ Department of Information Management, and Innovation Center for Big Data and Digital Convergence, Yuan Ze University, \\ Chung-Li 32003, Taiwan \\ ${ }^{4}$ Center for Fundamental Science, Kaohsiung Medical University, Kaohsiung 807, Taiwan
}

Correspondence should be addressed to Chin-Tzong Pang; imctpang@saturn.yzu.edu.tw

Received 4 May 2014; Accepted 31 July 2014; Published 31 August 2014

Academic Editor: Jong Kyu Kim

Copyright (c) $2014 \mathrm{Lu}$-Chuan Ceng et al. This is an open access article distributed under the Creative Commons Attribution License, which permits unrestricted use, distribution, and reproduction in any medium, provided the original work is properly cited.

\begin{abstract}
We introduce and analyze a hybrid steepest-descent algorithm by combining Korpelevich's extragradient method, the steepestdescent method, and the averaged mapping approach to the gradient-projection algorithm. It is proven that under appropriate assumptions, the proposed algorithm converges strongly to the unique solution of a triple hierarchical constrained optimization problem (THCOP) over the common fixed point set of finitely many nonexpansive mappings, with constraints of finitely many generalized mixed equilibrium problems (GMEPs), finitely many variational inclusions, and a convex minimization problem (CMP) in a real Hilbert space.
\end{abstract}

\section{Introduction}

Let $H$ be a real Hilbert space with inner product $\langle\cdot, \cdot\rangle$ and norm $\|\cdot\|$; let $C$ be a nonempty closed convex subset of $H$ and let $P_{C}$ be the metric projection of $H$ onto $C$. Let $S: C \rightarrow H$ be a nonlinear mapping on $C$. We denote by $\operatorname{Fix}(S)$ the set of fixed points of $S$ and by $\mathbf{R}$ the set of all real numbers. A mapping $S: C \rightarrow H$ is called $L$-Lipschitz continuous if there exists a constant $L>0$ such that

$$
\|S x-S y\| \leq L\|x-y\|, \quad \forall x, y \in C .
$$

In particular, if $L=1$ then $S$ is called a nonexpansive mapping; if $L \in(0,1)$ then $S$ is called a contraction.

Let $A: C \rightarrow H$ be a nonlinear mapping on $C$. The classical variational inequality problem (VIP) [1] is to find a point $x \in C$ such that

$$
\langle A x, y-x\rangle \geq 0, \quad \forall y \in C .
$$

The solution set of VIP (2) is denoted by $\operatorname{VI}(C, A)$.
In 1976, Korpelevich [2] proposed an iterative algorithm for solving the VIP (2) in Euclidean space $\mathbf{R}^{n}$ :

$$
\begin{array}{r}
y_{n}=P_{C}\left(x_{n}-\tau A x_{n}\right), \\
x_{n+1}=P_{C}\left(x_{n}-\tau A y_{n}\right), \\
\forall n \geq 0,
\end{array}
$$

with $\tau>0$ a given number, which is known as the extragradient method. See, for example, [3-7] and the references therein.

Let $\varphi: C \rightarrow \mathbf{R}$ be a real-valued function; let $A:$ $H \rightarrow H$ be a nonlinear mapping and let $\Theta: C \times C \rightarrow \mathbf{R}$ be a bifunction. In 2008, Peng and Yao [8] introduced the following generalized mixed equilibrium problem (GMEP) of finding $x \in C$ such that

$$
\Theta(x, y)+\varphi(y)-\varphi(x)+\langle A x, y-x\rangle \geq 0, \quad \forall y \in C .
$$

We denote the set of solutions of GMEP (4) by $\operatorname{GMEP}(\Theta, \varphi, A)$. 
In [8], Peng and Yao assumed that $\Theta: C \times C \rightarrow \mathbf{R}$ is a bifunction satisfying conditions (A1)-(A4) and $\varphi: C \rightarrow \mathbf{R}$ is a lower semicontinuous and convex function with restriction (B1) or (B2), where

(A1) $\Theta(x, x)=0$ for all $x \in C$;

(A2) $\Theta$ is monotone; that is, $\Theta(x, y)+\Theta(y, x) \leq 0$ for any $x, y \in C$;

(A3) $\Theta$ is upper-hemicontinuous; that is, for each $x, y, z \in$ $C$,

$$
\limsup _{t \rightarrow 0^{+}} \Theta(t z+(1-t) x, y) \leq \Theta(x, y)
$$

(A4) $\Theta(x, \cdot)$ is convex and lower semicontinuous for each $x \in C$;

(B1) for each $x \in H$ and $r>0$, there exists a bounded subset $D_{x} \subset C$ and $y_{x} \in C$ such that for any $z \in C \backslash D_{x}$,

$$
\Theta\left(z, y_{x}\right)+\varphi\left(y_{x}\right)-\varphi(z)+\frac{1}{r}\left\langle y_{x}-z, z-x\right\rangle<0
$$

(B2) $C$ is a bounded set.

Given a positive number $r>0$. Let $T_{r}^{(\Theta, \varphi)}: H \rightarrow C$ be the solution set of the auxiliary mixed equilibrium problem; that is, for each $x \in H$,

$$
\begin{aligned}
T_{r}^{(\Theta, \varphi)}(x):=\{y \in C: & \Theta(y, z)+\varphi(z)-\varphi(y) \\
+ & \left.\frac{1}{r}\langle y-x, z-y\rangle \geq 0, \forall z \in C\right\} .
\end{aligned}
$$

Let $f: C \rightarrow \mathbf{R}$ be a convex and continuously Fréchet differentiable functional. Consider the convex minimization problem (CMP) of minimizing $f$ over the constraint set $C$ :

$$
\min _{x \in C} f(x)
$$

(assuming the existence of minimizers). We denote by $\Gamma$ the set of minimizers of CMP (8).

On the other hand, let $B$ be a single-valued mapping of $C$ into $H$ and $R$ be a set-valued mapping with $D(R)=C$. Considering the following variational inclusion, find a point $x \in C$ such that

$$
0 \in B x+R x
$$

We denote by $I(B, R)$ the solution set of the variational inclusion (9). Let a set-valued mapping $R: D(R) \subset H \rightarrow$ $2^{H}$ be maximal monotone. We define the resolvent operator $J_{R, \lambda}: H \rightarrow \overline{D(R)}$ associated with $R$ and $\lambda$ as follows:

$$
J_{R, \lambda}=(I+\lambda R)^{-1}, \quad \forall x \in H,
$$

where $\lambda$ is a positive number.

Let $S$ and $T$ be two nonexpansive mappings. In 2009, Yao et al. [9] considered the following hierarchical VIP: find hierarchically a fixed point of $T$, which is a solution to the VIP for monotone mapping $I-S$; namely, find $\tilde{x} \in \operatorname{Fix}(T)$ such that

$$
\langle(I-S) \tilde{x}, p-\tilde{x}\rangle \geq 0, \quad \forall p \in \operatorname{Fix}(T) .
$$

The solution set of the hierarchical VIP (11) is denoted by $\Lambda$. It is not hard to check that solving the hierarchical VIP (11) is equivalent to the fixed point problem of the composite mapping $P_{\mathrm{Fix}(T)} S$; that is, find $\tilde{x} \in C$ such that $\tilde{x}=P_{\operatorname{Fix}(T)} S \tilde{x}$. The authors [9] introduced and analyzed the following iterative algorithm for solving the hierarchical VIP (11):

$$
\begin{array}{r}
y_{n}=\beta_{n} S x_{n}+\left(1-\beta_{n}\right) x_{n}, \\
x_{n+1}=\alpha_{n} V x_{n}+\left(1-\alpha_{n}\right) T y_{n}, \\
\forall n \geq 0 .
\end{array}
$$

In this paper, we introduce and study the following triple hierarchical constrained optimization problem (THCOP) with constraints of the CMP (8), finitely many GMEPs and finitely many variational inclusions.

Problem $I$. Let $M, N$, and $K$ be three positive integers. Assume that

(i) $f: C \rightarrow \mathbf{R}$ is a convex and continuously Fréchet differentiable functional with $L$-Lipschitz continuous gradient $\nabla f, S_{i}: H \rightarrow H$ is a nonexpansive mapping, and $A_{j}: H \rightarrow H$ is $\zeta_{j}$-inverse-strongly monotone for $i=1,2, \ldots, N$ and $j=1,2, \ldots, K$;

(ii) $\widetilde{A}_{1}: H \rightarrow H$ is $\alpha$-inverse strongly monotone and $\widetilde{A}_{2}: H \rightarrow H$ is $\beta$-strongly monotone and $\kappa$ Lipschitz continuous;

(iii) $\Theta_{j}$ is a bifunctions from $C \times C$ to $\mathbf{R}$ satisfying (A1)(A4), and $\varphi_{j}: C \rightarrow \mathbf{R}$ is a lower semicontinuous and convex functional with restriction (B1) or (B2) for $j=1,2, \ldots, K$;

(iv) $R_{k}: C \rightarrow 2^{H}$ is a maximal monotone mapping and $B_{k}: C \rightarrow H$ is $\eta_{k}$-inverse strongly monotone for $k=1,2, \ldots, M$;

(v) $\operatorname{VI}\left(\cap_{i=i}^{N} \operatorname{Fix}\left(S_{i}\right), \widetilde{A}_{1}\right) \neq \emptyset$ with $\left(\cap_{i=i}^{N} \operatorname{Fix}\left(S_{i}\right)\right) \subset\left(\cap_{j=1}^{K}\right.$ $\left.\operatorname{GMEP}\left(\Theta_{j}, \varphi_{j}, A_{j}\right)\right) \cap\left(\cap_{k=1}^{M} I\left(B_{k}, R_{k}\right)\right) \cap \Gamma$.

Then the objective is to

$$
\begin{aligned}
\text { find } x^{*} \in \mathrm{VI} & \left(\mathrm{VI}\left(\bigcap_{i=i}^{N} \operatorname{Fix}\left(S_{i}\right), \widetilde{A}_{1}\right), \widetilde{A}_{2}\right) \\
:= & \left\{x^{*} \in \mathrm{VI}\left(\bigcap_{i=i}^{N} \operatorname{Fix}\left(S_{i}\right), \widetilde{A}_{1}\right):\left\langle\widetilde{A}_{2} x^{*}, v-x^{*}\right\rangle\right. \\
& \left.\geq 0, \forall v \in \mathrm{VI}\left(\bigcap_{i=i}^{N} \operatorname{Fix}\left(S_{i}\right), \widetilde{A}_{1}\right)\right\} .
\end{aligned}
$$


Motivated and inspired by the above facts, we introduce and analyze a hybrid iterative algorithm via Korpelevich's extragradient method, the steepest-descent method, and the gradient-projection algorithm obtained by the averaged mapping approach. It is proven that under mild conditions, the proposed algorithm converges strongly to a unique element of $\operatorname{VI}\left(\operatorname{VI}\left(\cap_{i=1}^{N} \operatorname{Fix}\left(S_{i}\right), \widetilde{A}_{1}\right), \widetilde{A}_{2}\right)$ with $\left(\cap_{i=1}^{N} \operatorname{Fix}\left(S_{i}\right)\right) \subset$ $\left(\cap_{j=1}^{K} \operatorname{GMEP}\left(\Theta_{j}, \varphi_{j}, A_{j}\right)\right) \cap\left(\cap_{k=1}^{M} I\left(B_{k}, R_{k}\right)\right) \cap \Gamma$, that is, the unique solution of the THCOP (13). In this paper, the results we acquired improve and extend the existing results found in this field.

\section{Preliminaries}

Throughout this paper, we assume that $H$ is a real Hilbert space of which inner product and norm are denoted by $\langle\cdot, \cdot\rangle$ and $\|\cdot\|$, respectively. Let $C$ be a nonempty closed convex subset of $H$. We write $x_{n} \rightarrow x$ to indicate that the sequence $\left\{x_{n}\right\}$ converges weakly to $x$ and $x_{n} \rightarrow x$ to indicate that the sequence $\left\{x_{n}\right\}$ converges strongly to $x$. Moreover, we use $\omega_{w}\left(x_{n}\right)$ to denote the weak $\omega$-limit set of the sequence $\left\{x_{n}\right\}$; that is,

$$
\begin{aligned}
& \omega_{w}\left(x_{n}\right) \\
& :=\left\{x \in H: x_{n_{i}} \rightarrow x \text { for some subsequence }\left\{x_{n_{i}}\right\} \text { of }\left\{x_{n}\right\}\right\} .
\end{aligned}
$$

Definition 1. A mapping $A: C \rightarrow H$ is called

(i) monotone if

$$
\langle A x-A y, x-y\rangle \geq 0, \quad \forall x, y \in C ;
$$

(ii) $\eta$-strongly monotone if there exists a constant $\eta>0$ such that

$$
\langle A x-A y, x-y\rangle \geq \eta\|x-y\|^{2}, \quad \forall x, y \in C ;
$$

(iii) $\zeta$-inverse-strongly monotone if there exists a constant $\zeta>0$ such that

$$
\langle A x-A y, x-y\rangle \geq \zeta\|A x-A y\|^{2}, \quad \forall x, y \in C .
$$

It is obvious that if $A$ is $\zeta$-inverse-strongly monotone, then $A$ is monotone and $1 / \zeta$-Lipschitz continuous. Moreover, we also have that, for all $u, v \in C$ and $\lambda>0$,

$$
\begin{aligned}
\|(I- & \lambda A) u-(I-\lambda A) v \|^{2} \\
& \leq\|u-v\|^{2}+\lambda(\lambda-2 \zeta)\|A u-A v\|^{2} .
\end{aligned}
$$

So, if $\lambda \leq 2 \zeta$, then $I-\lambda A$ is a nonexpansive mapping from $C$ to $H$.

The metric projection from $H$ onto $C$ is the mapping $P_{C}$ : $H \rightarrow C$ which assigns to each point $x \in H$, the unique point $P_{C} x \in C$, satisfying the property

$$
\left\|x-P_{C} x\right\|=\inf _{y \in C}\|x-y\|=: d(x, C) .
$$

Some important properties of projections are gathered in the following proposition.

Proposition 2. For given $x \in H$ and $z \in C$ :

(i) $z=P_{C} x \Leftrightarrow\langle x-z, y-z\rangle \leq 0, \forall y \in C$;

(ii) $z=P_{C} x \Leftrightarrow\|x-z\|^{2} \leq\|x-y\|^{2}-\|y-z\|^{2}, \forall y \in C$;

(iii) $\left\langle P_{C} x-P_{C} y, x-y\right\rangle \geq\left\|P_{C} x-P_{C} y\right\|^{2}, \forall y \in H$. (This implies that $P_{C}$ is nonexpansive and monotone.)

Next we list some elementary conclusions for the mixed equilibrium problem where $\operatorname{MEP}(\Theta, \varphi)$ is the solution set.

Proposition 3 (see [10]). Assume that $\Theta: C \times C \rightarrow \mathbf{R}$ satisfies (A1)-(A4) and let $\varphi: C \rightarrow \mathbf{R}$ be a proper lower semicontinuous and convex function. Assume that either (B1) or (B2) holds. For $r>0$ and $x \in H$, define a mapping $T_{r}^{(\Theta, \varphi)}: H \rightarrow C$ as follows:

$$
\begin{aligned}
T_{r}^{(\Theta, \varphi)}(x)=\{z \in C: & \Theta(z, y)+\varphi(y)-\varphi(z) \\
+ & \left.\frac{1}{r}\langle y-z, z-x\rangle \geq 0, \forall y \in C\right\}
\end{aligned}
$$

for all $x \in H$. Then the following hold:

(i) for each $x \in H, T_{r}^{(\Theta, \varphi)}(x)$ is nonempty and singlevalued;

(ii) $T_{r}^{(\Theta, \varphi)}$ is firmly nonexpansive; that is, for any $x, y \in H$,

$$
\left\|T_{r}^{(\Theta, \varphi)} x-T_{r}^{(\Theta, \varphi)} y\right\|^{2} \leq\left\langle T_{r}^{(\Theta, \varphi)} x-T_{r}^{(\Theta, \varphi)} y, x-y\right\rangle ;
$$

(iii) $\operatorname{Fix}\left(T_{r}^{(\Theta, \varphi)}\right)=\operatorname{MEP}(\Theta, \varphi)$;

(iv) $\operatorname{MEP}(\Theta, \varphi)$ is closed and convex;

$$
\begin{gathered}
\text { (v) }\left\|T_{s}^{(\Theta, \varphi)} x-T_{t}^{(\Theta, \varphi)} x\right\|^{2} \leq \quad(s-t) / s\left\langle T_{s}^{(\Theta, \varphi)} x-\right. \\
\left.T_{t}^{(\Theta, \varphi)} x, T_{s}^{(\Theta, \varphi)} x-x\right\rangle \text { for all } s, t>0 \text { and } x \in H .
\end{gathered}
$$

In the following, we recall some facts and tools in a real Hilbert space $H$.

Lemma 4. Let $X$ be a real inner product space. Then there holds the following inequality

$$
\|x+y\|^{2} \leq\|x\|^{2}+2\langle y, x+y\rangle, \quad \forall x, y \in X .
$$

Lemma 5. Let $H$ be a real Hilbert space. Then the following hold:

(a) $\|x-y\|^{2}=\|x\|^{2}-\|y\|^{2}-2\langle x-y, y\rangle$ for all $x, y \in H$;

(b) $\|\lambda x+\mu y\|^{2}=\lambda\|x\|^{2}+\mu\|y\|^{2}-\lambda \mu\|x-y\|^{2}$ for all $x, y \epsilon$ $H$ and $\lambda, \mu \in[0,1]$ with $\lambda+\mu=1$;

(c) if $\left\{x_{n}\right\}$ is a sequence in $H$ such that $x_{n} \rightarrow x$, it follows that

$$
\begin{aligned}
& \limsup _{n \rightarrow \infty}\left\|x_{n}-y\right\|^{2} \\
& \quad=\limsup _{n \rightarrow \infty}\left\|x_{n}-x\right\|^{2}+\|x-y\|^{2}, \quad \forall y \in H .
\end{aligned}
$$


Definition 6. A mapping $T: H \rightarrow H$ is said to be an averaged mapping if it can be written as the average of the identity $I$ and a nonexpansive mapping; that is,

$$
T \equiv(1-\alpha) I+\alpha S
$$

where $\alpha \in(0,1)$ and $S: H \rightarrow H$ is nonexpansive. More precisely, when the last equality holds, we say that $T$ is $\alpha$ averaged. Thus firmly nonexpansive mappings (particularly, projections) are 1/2-averaged mappings.

Lemma 7 (see [11]). Let $T: H \rightarrow H$ be a given mapping.

(i) $T$ is nonexpansive if and only if the complement $I-T$ is $1 / 2$-ism.

(ii) If $T$ is $\nu$-ism, then for $\gamma>0, \gamma T$ is $\nu / \gamma$-ism.

(iii) $T$ is averaged if and only if the complement $I-T$ is $\nu$-ism for some $\nu>1 / 2$. Indeed, for $\alpha \in(0,1), T$ is $\alpha$-averaged if and only if $I-T$ is $1 / 2 \alpha$-ism.

Lemma 8 (see [11]). Let $S, T, V: H \rightarrow H$ be given operators.

(i) If $T=(1-\alpha) S+\alpha V$ for some $\alpha \in(0,1)$ and if $S$ is averaged and $V$ is nonexpansive, then $T$ is averaged.

(ii) $T$ is firmly nonexpansive if and only if the complement $I-T$ is firmly nonexpansive.

(iii) If $T=(1-\alpha) S+\alpha V$ for some $\alpha \in(0,1)$ and if $S$ is firmly nonexpansive and $V$ is nonexpansive, then $T$ is averaged.

(iv) The composite of finitely many averaged mappings is averaged. That is, if each of the mappings $\left\{T_{i}\right\}_{i=1}^{N}$ is averaged, then so is the composite $T_{1} \cdots T_{N}$. In particular, if $T_{1}$ is $\alpha_{1}$-averaged and $T_{2}$ is $\alpha_{2}$-averaged, where $\alpha_{1}, \alpha_{2} \in(0,1)$, then the composite $T_{1} T_{2}$ is $\alpha$ averaged, where $\alpha=\alpha_{1}+\alpha_{2}-\alpha_{1} \alpha_{2}$.

(v) If the mappings $\left\{T_{i}\right\}_{i=1}^{N}$ are averaged and have a common fixed point, then

$$
\bigcap_{i=1}^{N} \operatorname{Fix}\left(T_{i}\right)=\operatorname{Fix}\left(T_{1} T_{2} \cdots T_{N}\right) .
$$

The notation $\operatorname{Fix}(T)$ denotes the set of all fixed points of the mapping $T$; that is, $\operatorname{Fix}(T)=\{x \in H: T x=x\}$.

Let $f: C \rightarrow \mathbf{R}$ be a convex functional with $L$ Lipschitz continuous gradient $\nabla f$. It is well known that the gradient-projection algorithm (GPA) generates a sequence $\left\{x_{n}\right\}$ determined by the gradient $\nabla f$ and the metric projection $P_{C}$ :

$$
x_{n+1}:=P_{C}\left(x_{n}-\lambda \nabla f\left(x_{n}\right)\right), \quad \forall n \geq 0,
$$

or more generally,

$$
x_{n+1}:=P_{C}\left(x_{n}-\lambda_{n} \nabla f\left(x_{n}\right)\right), \quad \forall n \geq 0,
$$

where, in both (26) and (27), the initial guess $x_{0}$ is taken from $C$ arbitrarily, and the parameters $\lambda$ or $\lambda_{n}$ are positive real numbers. The convergence of algorithms (26) and (27) depends on the behavior of the gradient $\nabla f$.
Lemma 9 (see [12, Demiclosedness principle]). Let $C$ be a nonempty closed convex subset of a real Hilbert space $H$. Let $T$ be a nonexpansive self-mapping on $C$. Then $I-T$ is demiclosed. That is, whenever $\left\{x_{n}\right\}$ is a sequence in $C$ weakly converging to some $x \in C$ and the sequence $\left\{(I-T) x_{n}\right\}$ strongly converges to some $y$, it follows that $(I-T) x=y$. Here $I$ is the identity operator of $H$.

Lemma 10. Let $A: C \rightarrow H$ be a monotone mapping. In the context of the variational inequality problem the characterization of the projection (see Proposition 2(i)) implies

$$
u \in \mathrm{VI}(C, A) \Longleftrightarrow u=P_{C}(u-\lambda A u), \quad \lambda>0 .
$$

Let $C$ be a nonempty closed convex subset of a real Hilbert space $H$. We introduce some notations. Let $\lambda$ be a number in $(0,1]$ and let $\mu>0$. Associating with a nonexpansive mapping $T: C \rightarrow H$, we define the mapping $T^{\lambda}: C \rightarrow H$ by

$$
T^{\lambda} x:=T x-\lambda \mu F(T x), \quad \forall x \in C,
$$

where $F: H \rightarrow H$ is an operator such that, for some positive constants $\kappa, \eta>0, F$ is $\kappa$-Lipschitzian and $\eta$-strongly monotone on $H$; that is, $F$ satisfies the conditions:

$$
\|F x-F y\| \leq \kappa\|x-y\|, \quad\langle F x-F y, x-y\rangle \geq \eta\|x-y\|^{2}
$$

for all $x, y \in H$.

Lemma 11 (see [13, Lemma 3.1]). $T^{\lambda}$ is a contraction provided by $0<\mu<2 \eta / \kappa^{2}$; that is,

$$
\left\|T^{\lambda} x-T^{\lambda} y\right\| \leq(1-\lambda \tau)\|x-y\|, \quad \forall x, y \in C,
$$

where $\tau=1-\sqrt{1-\mu\left(2 \eta-\mu \kappa^{2}\right)} \in(0,1]$.

Lemma 12 (see [13]). Let $\left\{s_{n}\right\}$ be a sequence of nonnegative numbers satisfying the conditions

$$
s_{n+1} \leq\left(1-\alpha_{n}\right) s_{n}+\alpha_{n} \beta_{n}, \quad \forall n \geq 1,
$$

where $\left\{\alpha_{n}\right\}$ and $\left\{\beta_{n}\right\}$ are sequences of real numbers such that

(i) $\left\{\alpha_{n}\right\} \subset[0,1]$ and $\sum_{n=1}^{\infty} \alpha_{n}=\infty$, or equivalently,

$$
\prod_{n=1}^{\infty}\left(1-\alpha_{n}\right):=\lim _{n \rightarrow \infty} \prod_{k=1}^{n}\left(1-\alpha_{k}\right)=0 ;
$$

(ii) $\lim \sup _{n \rightarrow \infty} \beta_{n} \leq 0$, or $\sum_{n=1}^{\infty}\left|\alpha_{n} \beta_{n}\right|<\infty$.

Then $\lim _{n \rightarrow \infty} s_{n}=0$.

Recall that a Banach space $X$ is said to satisfy Opial's property [12] if, for any given sequence $\left\{x_{n}\right\} \subset X$ which converges weakly to an element $x \in X$, there holds the inequality

$$
\limsup _{n \rightarrow \infty}\left\|x_{n}-x\right\|<\limsup _{n \rightarrow \infty}\left\|x_{n}-y\right\|, \quad \forall y \in X, \quad y \neq x .
$$


It is well known that every Hilbert space $H$ satisfies Opial's property in [12].

Finally, recall that a set-valued mapping $T: D(T) \subset$ $H \rightarrow 2^{H}$ is called monotone if for all $x, y \in D(T), f \in T x$, and $g \in T y$ imply

$$
\langle f-g, x-y\rangle \geq 0 .
$$

A set-valued mapping $T$ is called maximal monotone if $T$ is monotone and $(I+\lambda T) D(T)=H$ for each $\lambda>0$, where $I$ is the identity mapping of $H$. We denote by $G(T)$ the graph of $T$. It is known that a monotone mapping $T$ is maximal if and only if, for $(x, f) \in H \times H,\langle f-g, x-y\rangle \geq 0$, for every $(y, g) \in G(T)$, implies $f \in T x$. Let $A: C \rightarrow H$ be a monotone, $k$-Lipschitzcontinuous mapping and let $N_{C} v$ be the normal cone to $C$ at $v \in C$; that is,

$$
N_{C} v=\{u \in H:\langle v-p, u\rangle \geq 0, \forall p \in C\} .
$$

Define

$$
\widetilde{T} v= \begin{cases}A v+N_{C} v, & \text { if } v \in C, \\ \emptyset, & \text { if } v \notin C .\end{cases}
$$

Then, $\widetilde{T}$ is maximal monotone such that

$$
0 \in \widetilde{T} v \Longleftrightarrow v \in \operatorname{VI}(C, A)
$$

Let $R: D(R) \subset H \rightarrow 2^{H}$ be a maximal monotone mapping. Let $\lambda, \mu>0$ be two positive numbers.

Lemma 13 (see [14]). There holds the resolvent identity

$$
J_{R, \lambda} x=J_{R, \mu}\left(\frac{\mu}{\lambda} x+\left(1-\frac{\mu}{\lambda}\right) J_{R, \lambda} x\right), \quad \forall x \in H
$$

For $\lambda, \mu>0$, there holds the following relation that

$$
\begin{aligned}
&\left\|J_{R, \lambda} x-J_{R, \mu} y\right\| \leq\|x-y\|+|\lambda-\mu| \\
& \times\left(\frac{1}{\lambda}\left\|J_{R, \lambda} x-y\right\|+\frac{1}{\mu}\left\|x-J_{R, \mu} y\right\|\right), \\
& \forall x, y \in H .
\end{aligned}
$$

Based on Huang [15], there holds the following property for the resolvent operator $J_{R, \lambda}: H \rightarrow \overline{D(R)}$.

Lemma 14. $J_{R, \lambda}$ is single-valued and firmly nonexpansive; that is,

$$
\left\langle J_{R, \lambda} x-J_{R, \lambda} y, x-y\right\rangle \geq\left\|J_{R, \lambda} x-J_{R, \lambda} y\right\|^{2}, \quad \forall x, y \in H .
$$

Consequently, $J_{R, \lambda}$ is nonexpansive and monotone.
Lemma 15 (see [16]). Let $R$ be a maximal monotone mapping with $D(R)=C$. Then for any given $\lambda>0, u \in$ $C$ is a solution of problem (10) if and only if $u \in C$ satisfies

$$
u=J_{R, \lambda}(u-\lambda B u)
$$

Lemma 16 (see [17]). Let $R$ be a maximal monotone mapping with $D(R)=C$ and let $B: C \rightarrow H$ be a strongly monotone, continuous, and single-valued mapping. Then, for each $z \in H$, the equation $z \in(B+\lambda R) x$ has a unique solution $x_{\lambda}$ for $\lambda>0$.

Lemma 17 (see [16]). Let $R$ be a maximal monotone mapping with $D(R)=C$ and let $B: C \rightarrow H$ be a monotone, continuous, and single-valued mapping. Then $(I+\lambda(R+B)) C=H$ for each $\lambda>0$. In this case, $R+B$ is maximal monotone.

\section{Main Results}

In this section, we will introduce and analyze a hybrid steepest-descent algorithm for finding a solution of the THCOP (13) with constraints of several problems: the CMP (8), finitely many GMEPs, and finitely many variational inclusions in a real Hilbert space. This algorithm is based on Korpelevich's extragradient method, the steepest-descent method, and the averaged mapping approach to the gradientprojection algorithm. We prove the strong convergence of the proposed algorithm to a unique solution of THCOP (13) under suitable conditions. Throughout this paper, let $\left\{S_{i}\right\}_{i=1}^{N}$ be $N$ nonexpansive mappings $S_{i}: H \rightarrow H$ with $N \geq 1$ an integer. We write $S_{[k]}:=S_{k \bmod N}$, for integer $k \geq 1$, with the mod function taking values in the set $\{1,2, \ldots, N\}$ (i.e., if $k=j N+q$ for some integers $j \geq 0$ and $0 \leq q<N$, then $T_{[k]}=N$ if $q=0$ and $T_{[k]}=q$ if $\left.1 \leq q<N\right)$.

The following is to state and prove the main result in this paper.

Theorem 18. Let $C$ be a nonempty closed convex subset of a real Hilbert space $H$ and let $f: C \rightarrow \mathbf{R}$ be a convex and continuously Fréchet differentiable functional with L-Lipschitz continuous gradient $\nabla f$. Let $M, N, K \geq 1$ be three integers. Let $\Theta_{j}$ be a bifunctions from $C \times C$ to $\mathbf{R}$ satisfying (A1)-(A4), $\varphi_{j}: C \rightarrow \mathbf{R}$ a lower semicontinuous and convex functional with restriction (B1) or (B2), and $A_{j}: H \rightarrow H \zeta_{j}$-inversestrongly monotone for $j=1,2, \ldots, K$. Let $R_{k}: C \rightarrow 2^{H}$ be a maximal monotone mapping and let $B_{k}: C \rightarrow H$ be $\eta_{k}$-inverse strongly monotone for $k=1,2, \ldots, M$. Let $\left\{S_{i}\right\}_{i=1}^{N}$ be a finite family of nonexpansive mappings on $H$. Let $\widetilde{A}_{1}: H \rightarrow H$ be $\alpha$-inverse strongly monotone and let $\widetilde{A}_{2}:$ $H \rightarrow H$ be $\beta$-strongly monotone and $\kappa$-Lipschitz continuous. Assume that $\operatorname{VI}\left(\cap_{i=1}^{N} \operatorname{Fix}\left(S_{i}\right), \widetilde{A}_{1}\right) \neq \emptyset$ with $\left(\cap_{i=1}^{N} \operatorname{Fix}\left(S_{i}\right)\right) \quad \subset$ $\left(\cap_{j=1}^{K} \operatorname{GMEP}\left(\Theta_{j}, \varphi_{j}, A_{j}\right)\right) \cap\left(\cap_{k=1}^{M} I\left(B_{k}, R_{k}\right)\right) \cap \Gamma$. Let $\mu \in$ $\left(0,2 \beta / \kappa^{2}\right),\left\{\alpha_{n}\right\}_{n=0}^{\infty} \subset(0,1],\left\{\rho_{n}\right\}_{n=0}^{\infty} \subset(0,2 \alpha],\left\{\lambda_{k, n}\right\}_{n=0}^{\infty} \subset$ $\left[a_{k}, b_{k}\right] \subset\left(0,2 \eta_{k}\right)$, and $\left\{r_{j, n}\right\}_{n=0}^{\infty} \subset\left[c_{j}, d_{j}\right] \subset\left(0,2 \zeta_{j}\right)$ where 
$j \in\{1,2, \ldots, K\}$ and $k \in\{1,2, \ldots, M\}$. For arbitrarily given $x_{0} \in H$, let $\left\{x_{n}\right\}$ be a sequence generated by

$$
\begin{aligned}
u_{n}= & T_{r_{K, n}}^{\left(\Theta_{K}, \varphi_{K}\right)}\left(I-r_{K, n} A_{K}\right) T_{r_{K-1, n}}^{\left(\Theta_{K-1}, \varphi_{K-1}\right)}\left(I-r_{K-1, n} A_{K-1}\right) \\
& \cdots T_{r_{1, n}}^{\left(\Theta_{1}, \varphi_{1}\right)}\left(I-r_{1, n} A_{1}\right) x_{n}, \\
v_{n}= & J_{R_{M}, \lambda_{M, n}}\left(I-\lambda_{M, n} B_{M}\right) J_{R_{M-1}, \lambda_{M-1, n}}\left(I-\lambda_{M-1, n} B_{M-1}\right) \\
& \cdots J_{R_{1}, \lambda_{1, n}}\left(I-\lambda_{1, n} B_{1}\right) u_{n}, \\
y_{n}= & S_{[n+1]}\left(I-\rho_{n} \widetilde{A}_{1}\right) T_{n} v_{n}, \\
x_{n+1}= & y_{n}-\mu \alpha_{n} \widetilde{A}_{2} y_{n}, \quad \forall n \geq 0,
\end{aligned}
$$

where $P_{C}\left(I-\lambda_{n} \nabla f\right)=s_{n} I+\left(1-s_{n}\right) T_{n}$ (here $T_{n}$ is nonexpansive and $s_{n}:=s_{n}\left(\lambda_{n}\right)=\left(2-\lambda_{n} L\right) / 4 \in(0,1 / 2)$ for each $\lambda_{n} \in$ $(0,2 / L))$. Assume that

$$
\begin{aligned}
\bigcap_{i=1}^{N} \operatorname{Fix}\left(S_{i}\right) & =\operatorname{Fix}\left(S_{1} S_{2} \cdots S_{N}\right) \\
& =\operatorname{Fix}\left(S_{N} S_{1} \cdots S_{N-1}\right) \\
& =\cdots=\operatorname{Fix}\left(S_{2} S_{3} \cdots S_{N} S_{1}\right)
\end{aligned}
$$

and that the following conditions are satisfied:

(i) $\lim _{n \rightarrow \infty} \alpha_{n}=0, \sum_{n=0}^{\infty} \alpha_{n}=\infty$ and $\rho_{n} \leq \alpha_{n}$ for all $n \geq 0$

(ii) $\lim _{n \rightarrow \infty}\left(\left|\alpha_{n}-\alpha_{n+N}\right| / \alpha_{n+N}\right)=0$ or $\sum_{n=0}^{\infty}\left|\alpha_{n}-\alpha_{n+N}\right|<$ $\infty$;

(iii) $\lim _{n \rightarrow \infty}\left(\left|s_{n}-s_{n+N}\right| / \alpha_{n+N}\right)=0$ or $\sum_{n=0}^{\infty}\left|s_{n}-s_{n+N}\right|<$ $\infty$;

(iv) $\lim _{n \rightarrow \infty}\left(\left|\rho_{n}-\rho_{n+N}\right| / \rho_{n+N}\right)=0$ or $\sum_{n=0}^{\infty}\left|\rho_{n}-\rho_{n+N}\right|<$ $\infty$;

(v) $\lim _{n \rightarrow \infty}\left(\left|\lambda_{k, n}-\lambda_{k, n+N}\right| /\left(\alpha_{n+N}\right)\right)=0$ or $\sum_{n=0}^{\infty} \mid \lambda_{k, n}-$ $\lambda_{k, n+N} \mid<\infty$ for $k=1,2, \ldots, M$;

(vi) $\lim _{n \rightarrow \infty}\left(\left|r_{j, n}-r_{j, n+N}\right| /\left(\alpha_{n+N}\right)\right)=0$ or $\sum_{n=0}^{\infty} \mid r_{j, n}-$ $r_{j, n+N} \mid<\infty$ for $j=1,2, \ldots, K$.

Then the following hold:

(a) $\left\{x_{n}\right\}_{n=0}^{\infty}$ is bounded;

(b) $\lim _{n \rightarrow \infty}\left\|x_{n}-x_{n+N}\right\|=0$;

(c) $\lim _{n \rightarrow \infty}\left\|x_{n}-S_{[n+N]} \cdots S_{[n+1]} x_{n}\right\|=0$ provided $\lim _{n \rightarrow \infty}\left(\left\|x_{n}-y_{n}\right\|+\left\|T_{n} v_{n}-v_{n}\right\|\right)=0$

(d) $\left\{x_{n}\right\}_{n=0}^{\infty}$ converges strongly to the unique element of $\operatorname{VI}\left(\operatorname{VI}\left(\cap_{i=1}^{N} \operatorname{Fix}\left(S_{i}\right), \widetilde{A}_{1}\right), \widetilde{A}_{2}\right)$ provided $\left\|x_{n}-y_{n}\right\|+$ $\left\|T_{n} v_{n}-v_{n}\right\|=o\left(\rho_{n}\right)$.

Proof. Let $\left\{x^{*}\right\}=\operatorname{VI}\left(\operatorname{VI}\left(\Omega, \widetilde{A}_{1}\right), \widetilde{A}_{2}\right)$. Since $\nabla f$ is $L$ Lipschitzian, it follows that $\nabla f$ is $1 / L$-ism. By Lemma 7 (ii), we know that for $\lambda>0, \lambda \nabla f$ is $1 / \lambda L$-ism. So by Lemma 7 (iii), we deduce that $I-\lambda \nabla f$ is $\lambda L / 2$-averaged. Now since the projection $P_{C}$ is $1 / 2$-averaged, it is easy to see from Lemma 8(iv) that the composite $P_{C}(I-\lambda \nabla f)$ is $(2+\lambda L) / 4$-averaged for $\lambda \epsilon$ $(0,2 / L)$. Hence we obtain that, for each $n \geq 0, P_{C}\left(I-\lambda_{n} \nabla f\right)$ is $\left(2+\lambda_{n} L\right) / 4$-averaged for each $\lambda_{n} \in(0,2 / L)$. Therefore, we can write

$$
\begin{aligned}
P_{C}\left(I-\lambda_{n} \nabla f\right) & =\frac{2-\lambda_{n} L}{4} I+\frac{2+\lambda_{n} L}{4} T_{n} \\
& =s_{n} I+\left(1-s_{n}\right) T_{n},
\end{aligned}
$$

where $T_{n}$ is nonexpansive and $s_{n}:=s_{n}\left(\lambda_{n}\right)=\left(2-\lambda_{n} L\right) / 4 \epsilon$ $(0,1 / 2)$ for each $\lambda_{n} \in(0,2 / L)$. Since $\widetilde{A}_{2}$ is $\kappa$-Lipschitz continuous, we get

$$
\left\|\widetilde{A}_{2} y_{n}-\widetilde{A}_{2} x^{*}\right\| \leq \kappa\left\|y_{n}-x^{*}\right\|, \quad \forall n \geq 0 .
$$

Putting $z_{n}=\left(I-\rho_{n} \widetilde{A}_{1}\right) T_{n} v_{n}$, for all $n \geq 0$, we have

$$
\begin{aligned}
x_{n+1} & =y_{n}-\mu \alpha_{n} \widetilde{A}_{2} y_{n} \\
& =S_{[n+1]} z_{n}-\mu \alpha_{n} \widetilde{A}_{2} S_{[n+1]} z_{n} \\
& =S_{[n+1]}^{\alpha_{n}} z_{n}, \quad \forall n \geq 0 .
\end{aligned}
$$

Put

$$
\begin{aligned}
\Delta_{n}^{j}= & T_{r_{j, n}}^{\left(\Theta_{j}, \varphi_{j}\right)}\left(I-r_{j, n} A_{j}\right) T_{r_{j-1, n}}^{\left(\Theta_{j-1}, \varphi_{j-1}\right)}\left(I-r_{j-1, n} A_{j-1}\right) \\
& \cdots T_{r_{1, n}}^{\left(\Theta_{1}, \varphi_{1}\right)}\left(I-r_{1, n} A_{1}\right) x_{n}
\end{aligned}
$$

for all $j \in\{1,2, \ldots, K\}$ and $n \geq 0$,

$$
\begin{aligned}
\Lambda_{n}^{k}= & J_{R_{k}, \lambda_{k, n}}\left(I-\lambda_{k, n} B_{k}\right) J_{R_{k-1}, \lambda_{k-1, n}}\left(I-\lambda_{k-1, n} B_{k-1}\right) \\
& \cdots J_{R_{1}, \lambda_{1, n}}\left(I-\lambda_{1, n} B_{1}\right)
\end{aligned}
$$

for all $k \in\{1,2, \ldots, M\}, \Delta_{n}^{0}=I$, and $\Lambda_{n}^{0}=I$, where $I$ is the identity mapping on $H$. Then we have that $u_{n}=\Delta_{n}^{K} x_{n}$ and $v_{n}=\Lambda_{n}^{M} u_{n}$.

We divide the rest of the proof into several steps.

Step 1. We prove that $\left\{x_{n}\right\}$ is bounded.

Indeed, utilizing (18) and Proposition 3(ii), we have

$$
\begin{aligned}
& \left\|u_{n}-x^{*}\right\| \\
& =\| T_{r_{K, n}^{\left(\Theta_{K}, \varphi_{K}\right)}}\left(I-r_{K, n} B_{K}\right) \Delta_{n}^{K-1} x_{n} \\
& \quad \quad-T_{r_{K, n}^{\left(\Theta_{K}, \varphi_{K}\right)}\left(I-r_{K, n} B_{K}\right) \Delta_{n}^{K-1} x^{*} \|} \\
& \leq\left\|\left(I-r_{K, n} B_{K}\right) \Delta_{n}^{K-1} x_{n}-\left(I-r_{K, n} B_{K}\right) \Delta_{n}^{K-1} x^{*}\right\| \\
& \leq\left\|\Delta_{n}^{K-1} x_{n}-\Delta_{n}^{K-1} x^{*}\right\| \\
& \vdots \\
& \leq\left\|\Delta_{n}^{0} x_{n}-\Delta_{n}^{0} x^{*}\right\| \\
& =\left\|x_{n}-x^{*}\right\| .
\end{aligned}
$$


Utilizing (18) and Lemma 14 we have

$$
\begin{aligned}
& \left\|v_{n}-x^{*}\right\| \\
= & \| J_{R_{M}, \lambda_{M, n}}\left(I-\lambda_{M, n} A_{M}\right) \Lambda_{n}^{M-1} u_{n} \\
& -J_{R_{M}, \lambda_{M, n}}\left(I-\lambda_{M, n} A_{M}\right) \Lambda_{n}^{M-1} x^{*} \| \\
\leq & \left\|\left(I-\lambda_{M, n} A_{M}\right) \Lambda_{n}^{M-1} u_{n}-\left(I-\lambda_{M, n} A_{M}\right) \Lambda_{n}^{M-1} x^{*}\right\| \\
\leq & \left\|\Lambda_{n}^{M-1} u_{n}-\Lambda_{n}^{M-1} x^{*}\right\| \\
& \vdots \\
\leq & \left\|\Lambda_{n}^{0} u_{n}-\Lambda_{n}^{0} x^{*}\right\| \\
= & \left\|u_{n}-x^{*}\right\| .
\end{aligned}
$$

Combining (50) and (51), we have

$$
\left\|v_{n}-x^{*}\right\| \leq\left\|x_{n}-x^{*}\right\| .
$$

Since $\widetilde{A}_{1}$ is $\alpha$-inverse strongly monotone and $\left\{\rho_{n}\right\}_{n=0}^{\infty} \quad C$ $(0,2 \alpha]$, we have

$$
\begin{aligned}
& \left\|T_{n} v_{n}-x^{*}-\rho_{n}\left(\widetilde{A}_{1} T_{n} v_{n}-\widetilde{A}_{1} x^{*}\right)\right\|^{2} \\
& =\left\|T_{n} v_{n}-x^{*}\right\|^{2} \\
& \quad-2 \rho_{n}\left\langle\widetilde{A}_{1} T_{n} v_{n}-\widetilde{A}_{1} x^{*}, T_{n} v_{n}-x^{*}\right\rangle \\
& \quad+\rho_{n}^{2}\left\|\widetilde{A}_{1} T_{n} v_{n}-\widetilde{A}_{1} x^{*}\right\|^{2} \\
& \leq\left\|T_{n} v_{n}-x^{*}\right\|^{2}-\rho_{n}\left(2 \alpha-\rho_{n}\right)\left\|\widetilde{A}_{1} T_{n} v_{n}-\widetilde{A}_{1} x^{*}\right\|^{2} \\
& \leq\left\|T_{n} v_{n}-x^{*}\right\|^{2} \\
& \leq\left\|v_{n}-x^{*}\right\|^{2} .
\end{aligned}
$$

Utilizing Lemma 11, we deduce from (52), $\rho_{n} \leq \alpha_{n}$, and $S_{[n+1]}^{\alpha_{n}} x^{*}=x^{*}-\alpha_{n} \mu \widetilde{A}_{2} x^{*}$ that for all $n \geq 0$

$$
\begin{aligned}
& \left\|x_{n+1}-x^{*}\right\| \\
& =\left\|S_{[n+1]}^{\alpha_{n}} z_{n}-x^{*}\right\| \\
& \leq\left\|S_{[n+1]}^{\alpha_{n}} z_{n}-S_{[n+1]}^{\alpha_{n}} x^{*}\right\|+\left\|S_{[n+1]}^{\alpha_{n}} x^{*}-x^{*}\right\| \\
& \leq\left(1-\alpha_{n} \tau\right)\left\|z_{n}-x^{*}\right\|+\alpha_{n} \mu\left\|\widetilde{A}_{2} x^{*}\right\| \\
& =\left(1-\alpha_{n} \tau\right)\left\|\left(I-\rho_{n} \widetilde{A}_{1}\right) T_{n} v_{n}-x^{*}\right\|+\alpha_{n} \mu\left\|\widetilde{A}_{2} x^{*}\right\|
\end{aligned}
$$

$$
\begin{aligned}
= & \left(1-\alpha_{n} \tau\right)\left\|T_{n} v_{n}-x^{*}-\rho_{n}\left(\widetilde{A}_{1} T_{n} v_{n}-\widetilde{A}_{1} x^{*}\right)-\rho_{n} \widetilde{A}_{1} x^{*}\right\| \\
& +\alpha_{n} \mu\left\|\widetilde{A}_{2} x^{*}\right\| \\
\leq & \left(1-\alpha_{n} \tau\right)\left[\left\|T_{n} v_{n}-x^{*}-\rho_{n}\left(\widetilde{A}_{1} T_{n} v_{n}-\widetilde{A}_{1} x^{*}\right)\right\|\right. \\
& \left.\quad+\rho_{n}\left\|\widetilde{A}_{1} x^{*}\right\|\right]+\alpha_{n} \mu\left\|\widetilde{A}_{2} x^{*}\right\| \\
\leq & \left(1-\alpha_{n} \tau\right)\left[\left\|v_{n}-x^{*}\right\|+\rho_{n}\left\|\widetilde{A}_{1} x^{*}\right\|\right]+\alpha_{n} \mu\left\|\widetilde{A}_{2} x^{*}\right\| \\
\leq & \left(1-\alpha_{n} \tau\right)\left[\left\|x_{n}-x^{*}\right\|+\rho_{n}\left\|\widetilde{A}_{1} x^{*}\right\|\right]+\alpha_{n} \mu\left\|\widetilde{A}_{2} x^{*}\right\| \\
\leq & \left(1-\alpha_{n} \tau\right)\left\|x_{n}-x^{*}\right\|+\rho_{n}\left\|\widetilde{A}_{1} x^{*}\right\|+\alpha_{n} \mu\left\|\widetilde{A}_{2} x^{*}\right\| \\
\leq & \left(1-\alpha_{n} \tau\right)\left\|x_{n}-x^{*}\right\|+\alpha_{n}\left\|\widetilde{A}_{1} x^{*}\right\| \\
& +\alpha_{n} \mu\left\|\widetilde{A}_{2} x^{*}\right\| \\
= & \left(1-\alpha_{n} \tau\right)\left\|x_{n}-x^{*}\right\| \\
& +\alpha_{n} \tau \frac{\left\|\widetilde{A}_{1} x^{*}\right\|+\mu\left\|\widetilde{A}_{2} x^{*}\right\|}{\tau} \\
\leq & \max \left\{\left\|x_{n}-x^{*}\right\|, \frac{\left\|\widetilde{A}_{1} x^{*}\right\|+\mu\left\|\widetilde{A}_{2} x^{*}\right\|}{\tau}\right\},
\end{aligned}
$$

where $\tau=1-\sqrt{1-\mu\left(2 \beta-\mu \kappa^{2}\right)}$. So, by induction we obtain

$$
\begin{aligned}
& \left\|x_{n}-x^{*}\right\| \\
& \leq \max \left\{\left\|x_{0}-x^{*}\right\|, \frac{\left\|\widetilde{A}_{1} x^{*}\right\|+\mu\left\|\widetilde{A}_{2} x^{*}\right\|}{\tau}\right\}, \quad \forall n \geq 0 .
\end{aligned}
$$

Hence $\left\{x_{n}\right\}_{n=0}^{\infty}$ is bounded. Since $\widetilde{A}_{1}: H \rightarrow H$ is $\alpha$-inverse strongly monotone, it is known that $\widetilde{A}_{1}$ is $1 / \alpha$-Lipschitz continuous. Thus, from (52), we get

$$
\begin{aligned}
\left\|\widetilde{A}_{1} T_{n} v_{n}-\widetilde{A}_{1} x^{*}\right\| & \leq \frac{1}{\alpha}\left\|T_{n} v_{n}-x^{*}\right\| \leq \frac{1}{\alpha}\left\|v_{n}-x^{*}\right\| \\
& \leq \frac{1}{\alpha}\left\|x_{n}-x^{*}\right\|, \quad \forall n \geq 0 .
\end{aligned}
$$

Consequently, the boundedness of $\left\{x_{n}\right\}$ ensures the boundedness of $\left\{v_{n}\right\},\left\{T_{n} v_{n}\right\}$, and $\left\{\widetilde{A}_{1} T_{n} v_{n}\right\}$. From $y_{n}=S_{[n+1]}(I-$ $\left.\rho_{n} \widetilde{A}_{1}\right) T_{n} v_{n}$ and the nonexpansivity of $S_{[n+1]}$, it follows that $\left\{y_{n}\right\}$ is bounded. Since $\widetilde{A}_{2}$ is $\kappa$-Lipschitz continuous, $\left\{\widetilde{A}_{2} y_{n}\right\}$ is also bounded.

Step 2. We prove that $\lim _{n \rightarrow \infty}\left\|x_{n}-x_{n+N}\right\|=0$. 
Indeed, utilizing (18) and (40), we obtain that

$$
\begin{aligned}
& \left\|v_{n+N}-v_{n}\right\| \\
& =\left\|\Lambda_{n+N}^{M} u_{n+N}-\Lambda_{n}^{M} u_{n}\right\| \\
& =\| J_{R_{M}, \lambda_{M, n+N}}\left(I-\lambda_{M, n+N} B_{M}\right) \Lambda_{n+N}^{M-1} u_{n+N} \\
& -J_{R_{M}, \lambda_{M, n}}\left(I-\lambda_{M, n} B_{M}\right) \Lambda_{n}^{M-1} u_{n} \| \\
& \leq \| J_{R_{M}, \lambda_{M, n+N}}\left(I-\lambda_{M, n+N} B_{M}\right) \Lambda_{n+N}^{M-1} u_{n+N} \\
& -J_{R_{M}, \lambda_{M, n+N}}\left(I-\lambda_{M, n} B_{M}\right) \Lambda_{n+N}^{M-1} u_{n+N} \| \\
& +\| J_{R_{M}, \lambda_{M, n+N}}\left(I-\lambda_{M, n} B_{M}\right) \Lambda_{n+N}^{M-1} u_{n+N} \\
& -J_{R_{M}, \lambda_{M, n}}\left(I-\lambda_{M, n} B_{M}\right) \Lambda_{n}^{M-1} u_{n} \| \\
& \leq\left\|\left(I-\lambda_{M, n+N} B_{M}\right) \Lambda_{n+N}^{M-1} u_{n+N}-\left(I-\lambda_{M, n} B_{M}\right) \Lambda_{n+N}^{M-1} u_{n+N}\right\| \\
& +\left\|\left(I-\lambda_{M, n} B_{M}\right) \Lambda_{n+N}^{M-1} u_{n+N}-\left(I-\lambda_{M, n} B_{M}\right) \Lambda_{n}^{M-1} u_{n}\right\| \\
& +\left|\lambda_{M, n+N}-\lambda_{M, n}\right| \\
& \times\left(\frac{1}{\lambda_{M, n+N}} \| J_{R_{M}, \lambda_{M, n+N}}\left(I-\lambda_{M, n} B_{M}\right) \Lambda_{n+N}^{M-1} u_{n+N}\right. \\
& -\left(I-\lambda_{M, n} B_{M}\right) \Lambda_{n}^{M-1} u_{n} \| \\
& +\frac{1}{\lambda_{M, n}} \|\left(I-\lambda_{M, n} B_{M}\right) \Lambda_{n+N}^{M-1} u_{n+N} \\
& \left.-J_{R_{M}, \lambda_{M, n}}\left(I-\lambda_{M, n} B_{M}\right) \Lambda_{n}^{M-1} u_{n} \|\right) \\
& \leq\left|\lambda_{M, n+N}-\lambda_{M, n}\right|\left(\left\|B_{M} \Lambda_{n+N}^{M-1} u_{n+N}\right\|+\widetilde{M}\right) \\
& +\left\|\Lambda_{n+N}^{M-1} u_{n+N}-\Lambda_{n}^{M-1} u_{n}\right\| \\
& \leq\left|\lambda_{M, n+N}-\lambda_{M, n}\right|\left(\left\|B_{M} \Lambda_{n+N}^{M-1} u_{n+N}\right\|+\widetilde{M}\right) \\
& +\left|\lambda_{M-1, n+N}-\lambda_{M-1, n}\right|\left(\left\|B_{M-1} \Lambda_{n+N}^{M-2} u_{n+N}\right\|+\widetilde{M}\right) \\
& +\left\|\Lambda_{n+N}^{M-2} u_{n+N}-\Lambda_{n}^{M-2} u_{n}\right\| \\
& \vdots \\
& \leq\left|\lambda_{M, n+N}-\lambda_{M, n}\right|\left(\left\|B_{M} \Lambda_{n+N}^{M-1} u_{n+N}\right\|+\widetilde{M}\right) \\
& +\left|\lambda_{M-1, n+N}-\lambda_{M-1, n}\right|\left(\left\|B_{M-1} \Lambda_{n+N}^{M-2} u_{n+N}\right\|+\widetilde{M}\right) \\
& +\cdots+\left|\lambda_{1, n+N}-\lambda_{1, n}\right|\left(\left\|B_{1} \Lambda_{n+N}^{0} u_{n+N}\right\|+\widetilde{M}\right) \\
& +\left\|\Lambda_{n+N}^{0} u_{n+N}-\Lambda_{n}^{0} u_{n}\right\| \\
& \leq \widetilde{M}_{0} \sum_{k=1}^{M}\left|\lambda_{k, n+N}-\lambda_{k, n}\right|+\left\|u_{n+N}-u_{n}\right\|,
\end{aligned}
$$

where

$$
\begin{gathered}
\sup _{n \geq 0,1 \leq i \leq M}\left\{\frac{1}{\lambda_{i, n+N}} \| J_{R_{i}, \lambda_{i, n+N}}\left(I-\lambda_{i, n} B_{i}\right) \Lambda_{n+N}^{i-1} u_{n+N}\right. \\
-\left(I-\lambda_{i, n} B_{i}\right) \Lambda_{n}^{i-1} u_{n} \| \\
+\frac{1}{\lambda_{i, n}} \|\left(I-\lambda_{i, n} B_{i}\right) \Lambda_{n+N}^{i-1} u_{n+N} \\
\left.\quad-J_{R_{i}, \lambda_{i, n}}\left(I-\lambda_{i, n} B_{i}\right) \Lambda_{n}^{i-1} u_{n} \|\right\} \leq \widetilde{M},
\end{gathered}
$$

for some $\widetilde{M}>0$ and $\sup _{n \geq 0}\left\{\sum_{k=1}^{M}\left\|B_{k} \Lambda_{n+N}^{k-1} u_{n+N}\right\|+\widetilde{M}\right\} \leq \widetilde{M}_{0}$ for some $\widetilde{M}_{0}>0$.

Furthermore, since $\nabla f$ is $1 / L$-ism, $P_{C}\left(I-\lambda_{n} \nabla f\right)$ is nonexpansive for $\lambda_{n} \in(0,2 / L)$. So, it follows that

$$
\begin{aligned}
\left\|P_{C}\left(I-\lambda_{n+N} \nabla f\right) v_{n}\right\| \\
\quad \leq\left\|P_{C}\left(I-\lambda_{n+N} \nabla f\right) v_{n}-x^{*}\right\|+\left\|x^{*}\right\| \\
\quad=\left\|P_{C}\left(I-\lambda_{n+N} \nabla f\right) v_{n}-P_{C}\left(I-\lambda_{n+N} \nabla f\right) x^{*}\right\|+\left\|x^{*}\right\| \\
\quad \leq\left\|v_{n}-x^{*}\right\|+\left\|x^{*}\right\| \\
\quad \leq\left\|v_{n}\right\|+2\left\|x^{*}\right\| .
\end{aligned}
$$

With the boundedness of $\left\{v_{n}\right\}$, this implies that $\left\{P_{C}(I-\right.$ $\left.\left.\lambda_{n+N} \nabla f\right) v_{n}\right\}$ is bounded. Also, observe that

$$
\begin{aligned}
& \left\|T_{n+N} v_{n}-T_{n} v_{n}\right\| \\
& =\| \frac{4 P_{C}\left(I-\lambda_{n+N} \nabla f\right)-\left(2-\lambda_{n+N} L\right) I}{2+\lambda_{n+N} L} v_{n} \\
& \quad-\frac{4 P_{C}\left(I-\lambda_{n} \nabla f\right)-\left(2-\lambda_{n} L\right) I}{2+\lambda_{n} L} v_{n} \| \\
& \leq\left\|\frac{4 P_{C}\left(I-\lambda_{n+N} \nabla f\right)}{2+\lambda_{n+N} L} v_{n}-\frac{4 P_{C}\left(I-\lambda_{n} \nabla f\right)}{2+\lambda_{n} L} v_{n}\right\| \\
& +\left\|\frac{2-\lambda_{n} L}{2+\lambda_{n} L} v_{n}-\frac{2-\lambda_{n+N} L}{2+\lambda_{n+N} L} v_{n}\right\| \\
& =\|\left(4\left(2+\lambda_{n} L\right) P_{C}\left(I-\lambda_{n+N} \nabla f\right) v_{n}\right. \\
& \left.\quad-4\left(2+\lambda_{n+N} L\right) P_{C}\left(I-\lambda_{n} \nabla f\right) v_{n}\right) \\
& \quad \times\left(\left(2+\lambda_{n+N} L\right)\left(2+\lambda_{n} L\right)\right)^{-1} \| \\
& +\frac{4 L\left|\lambda_{n+N}-\lambda_{n}\right|}{\left(2+\lambda_{n+N} L\right)\left(2+\lambda_{n} L\right)}\left\|v_{n}\right\|
\end{aligned}
$$




$$
\begin{aligned}
&=\|\left(4 L\left(\lambda_{n}-\lambda_{n+N}\right) P_{C}\left(I-\lambda_{n+N} \nabla f\right) v_{n}+4\left(2+\lambda_{n+N} L\right)\right. \\
&\left.\times\left(P_{C}\left(I-\lambda_{n+N} \nabla f\right) v_{n}-P_{C}\left(I-\lambda_{n} \nabla f\right) v_{n}\right)\right) \\
& \times\left(\left(2+\lambda_{n+N} L\right)\left(2+\lambda_{n} L\right)\right)^{-1} \| \\
&+\frac{4 L\left|\lambda_{n+N}-\lambda_{n}\right|}{\left(2+\lambda_{n+N} L\right)\left(2+\lambda_{n} L\right)}\left\|v_{n}\right\| \\
& \leq \frac{4 L\left|\lambda_{n}-\lambda_{n+N}\right|\left\|P_{C}\left(I-\lambda_{n+N} \nabla f\right) v_{n}\right\|}{\left(2+\lambda_{n+N} L\right)\left(2+\lambda_{n} L\right)} \\
&+\left(4\left(2+\lambda_{n+N} L\right)\right. \\
&\left.\times\left\|P_{C}\left(I-\lambda_{n+N} \nabla f\right) v_{n}-P_{C}\left(I-\lambda_{n} \nabla f\right) v_{n}\right\|\right) \\
& \times\left(\left(2+\lambda_{n+N} L\right)\left(2+\lambda_{n} L\right)\right)^{-1} \\
&+\frac{4 L\left|\lambda_{n+N}-\lambda_{n}\right|}{\left(2+\lambda_{n+N} L\right)\left(2+\lambda_{n} L\right)}\left\|v_{n}\right\| \\
& \leq\left|\lambda_{n+N}-\lambda_{n}\right|\left[L\left\|P_{C}\left(I-\lambda_{n+N} \nabla f\right) v_{n}\right\|\right. \\
&\left.\quad+4\left\|\nabla f\left(v_{n}\right)\right\|+L\left\|v_{n}\right\|\right] \\
& \leq \widetilde{M}_{1}\left|\lambda_{n+N}-\lambda_{n}\right|
\end{aligned}
$$

where $\sup _{n \geq 0}\left\{L\left\|P_{C}\left(I-\lambda_{n+N} \nabla f\right) v_{n}\right\|+4\left\|\nabla f\left(v_{n}\right)\right\|+L\left\|v_{n}\right\|\right\} \leq \widetilde{M}_{1}$ for some $\widetilde{M}_{1}>0$. Thus, we conclude from (57) and (60) that

$$
\begin{aligned}
& \left\|T_{n+N} v_{n+N}-T_{n} v_{n}\right\| \\
& \leq\left\|T_{n+N} v_{n+N}-T_{n+N} v_{n}\right\|+\left\|T_{n+N} v_{n}-T_{n} v_{n}\right\| \\
& \leq\left\|v_{n+N}-v_{n}\right\|+\widetilde{M}_{1}\left|\lambda_{n+N}-\lambda_{n}\right| \\
& \leq\left\|v_{n+N}-v_{n}\right\|+\frac{4 \widetilde{M}_{1}}{L}\left|s_{n+N}-s_{n}\right| \\
& \leq \widetilde{M}_{0} \sum_{k=1}^{M}\left|\lambda_{k, n+N}-\lambda_{k, n}\right|+\left\|u_{n+N}-u_{n}\right\| \\
& \quad+\frac{4 \widetilde{M}_{1}}{L}\left|s_{n+N}-s_{n}\right| .
\end{aligned}
$$

Also, utilizing Proposition 3(ii), (v), we deduce that

$$
\begin{aligned}
& \left\|u_{n+N}-u_{n}\right\| \\
= & \left\|\Delta_{n+N}^{K} x_{n+N}-\Delta_{n}^{K} x_{n}\right\| \\
= & \| T_{r_{K, n+N}^{\left(\Theta_{K}, \varphi_{K}\right)}}\left(I-r_{K, n+N} A_{K}\right) \Delta_{n+N}^{K-1} x_{n+N} \\
& \quad-T_{r_{K, n}}^{\left(\Theta_{K}, \varphi_{K}\right)}\left(I-r_{K, n} A_{K}\right) \Delta_{n}^{K-1} x_{n} \| \\
\leq & \| T_{r_{K, n+N}}^{\left(\Theta_{K}, \varphi_{K}\right)}\left(I-r_{K, n+N} A_{K}\right) \Delta_{n+N}^{K-1} x_{n+N}
\end{aligned}
$$

$$
\begin{aligned}
& -T_{r_{K, n}}^{\left(\Theta_{K}, \varphi_{K}\right)}\left(I-r_{K, n} A_{K}\right) \Delta_{n+N}^{K-1} x_{n+N} \| \\
& +\| T_{r_{K, n}}^{\left(\Theta_{K}, \varphi_{K}\right)}\left(I-r_{K, n} A_{K}\right) \Delta_{n+N}^{K-1} x_{n+N} \\
& -T_{r_{K, n}}^{\left(\Theta_{K}, \varphi_{K}\right)}\left(I-r_{K, n} A_{K}\right) \Delta_{n}^{K-1} x_{n} \|
\end{aligned}
$$

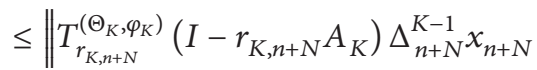

$$
\begin{aligned}
& -T_{r_{K, n}}^{\left(\Theta_{K}, \varphi_{K}\right)}\left(I-r_{K, n+N} A_{K}\right) \Delta_{n+N}^{K-1} x_{n+N} \| \\
& +\| T_{r_{K, n}}^{\left(\Theta_{K}, \varphi_{K}\right)}\left(I-r_{K, n+N} A_{K}\right) \Delta_{n+N}^{K-1} x_{n+N} \\
& -T_{r_{K, n}}^{\left(\Theta_{K}, \varphi_{K}\right)}\left(I-r_{K, n} A_{K}\right) \Delta_{n+N}^{K-1} x_{n+N} \| \\
& +\left\|\left(I-r_{K, n} A_{K}\right) \Delta_{n+N}^{K-1} x_{n+N}-\left(I-r_{K, n} A_{K}\right) \Delta_{n}^{K-1} x_{n}\right\| \\
& \leq \frac{\left|r_{K, n+N}-r_{K, n}\right|}{r_{K, n+N}} \\
& \times \| T_{r_{K, n+N}}^{\left(\Theta_{K}, \varphi_{K}\right)}\left(I-r_{K, n+N} A_{K}\right) \Delta_{n+N}^{K-1} x_{n+N} \\
& -\left(I-r_{K, n+N} A_{K}\right) \Delta_{n+N}^{K-1} x_{n+N} \| \\
& +\left|r_{K, n+N}-r_{K, n}\right|\left\|A_{K} \Delta_{n+N}^{K-1} x_{n+N}\right\| \\
& +\left\|\Delta_{n+N}^{K-1} x_{n+N}-\Delta_{n}^{K-1} x_{n}\right\| \\
& =\left|r_{K, n+N}-r_{K, n}\right|\left[\left\|A_{K} \Delta_{n+N}^{K-1} x_{n+N}\right\|+\frac{1}{r_{K, n+N}}\right. \\
& \times \| T_{r_{K, n+N}}^{\left(\Theta_{K}, \varphi_{K}\right)}\left(I-r_{K, n+N} A_{K}\right) \Delta_{n+N}^{K-1} x_{n+N} \\
& \left.-\left(I-r_{K, n+N} A_{K}\right) \Delta_{n+N}^{K-1} x_{n+N} \|\right] \\
& +\left\|\Delta_{n+N}^{K-1} x_{n+N}-\Delta_{n}^{K-1} x_{n}\right\| \\
& \leq\left|r_{K, n+N}-r_{K, n}\right|\left[\left\|A_{K} \Delta_{n+N}^{K-1} x_{n+N}\right\|+\frac{1}{r_{K, n+N}}\right. \\
& \times \| T_{r_{K, n+N}}^{\left(\Theta_{K}, \varphi_{K}\right)}\left(I-r_{K, n+N} A_{K}\right) \Delta_{n+N}^{K-1} x_{n+N} \\
& \left.-\left(I-r_{K, n+N} A_{K}\right) \Delta_{n+N}^{K-1} x_{n+N} \|\right] \\
& +\cdots+\left|r_{1, n+N}-r_{1, n}\right| \\
& \times\left[\left\|A_{1} \Delta_{n+N}^{0} x_{n+N}\right\|+\frac{1}{r_{1, n+N}}\right.
\end{aligned}
$$

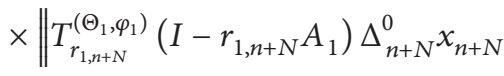

$$
\begin{aligned}
& \left.-\left(I-r_{1, n+N} A_{1}\right) \Delta_{n+N}^{0} x_{n+N} \|\right]
\end{aligned}
$$




$$
\begin{aligned}
& +\left\|\Delta_{n+N}^{0} x_{n+N}-\Delta_{n}^{0} x_{n}\right\| \\
\leq & \widetilde{M}_{2} \sum_{j=1}^{K}\left|r_{j, n+N}-r_{j, n}\right|+\left\|x_{n+N}-x_{n}\right\|,
\end{aligned}
$$

where $\widetilde{M}_{2}>0$ is a constant such that for each $n \geq 0$

$$
\begin{aligned}
& \sum_{j=1}^{K}\left[\left\|A_{j} \Delta_{n+N}^{j-1} x_{n+N}\right\|\right. \\
& +\frac{1}{r_{j, n+N}} \| T_{r_{j, n+N}^{\left(\Theta_{j}, \varphi_{j}\right)}}\left(I-r_{j, n+N} A_{j}\right) \Delta_{n+N}^{j-1} x_{n+N} \\
& \left.-\left(I-r_{j, n+N} A_{j}\right) \Delta_{n+N}^{j-1} x_{n+N} \|\right] \leq \widetilde{M}_{2} .
\end{aligned}
$$

Therefore, it follows from (18), (61), (62), and $\left\{\rho_{n}\right\}_{n=0}^{\infty} \subset(0,2 \alpha]$ that

$$
\begin{aligned}
\| & z_{n+N}-z_{n} \| \\
= & \left\|\left(T_{n+N} v_{n+N}-\rho_{n+N} \widetilde{A}_{1} T_{n+N} v_{n+N}\right)-\left(T_{n} v_{n}-\rho_{n} \widetilde{A}_{1} T_{n} v_{n}\right)\right\| \\
\leq & \|\left(T_{n+N} v_{n+N}-\rho_{n+N} \widetilde{A}_{1} T_{n+N} v_{n+N}\right) \\
& \quad-\left(T_{n} v_{n}-\rho_{n+N} \widetilde{A}_{1} T_{n} v_{n}\right) \| \\
& +\left\|\left(T_{n} v_{n}-\rho_{n+N} \widetilde{A}_{1} T_{n} v_{n}\right)-\left(T_{n} v_{n}-\rho_{n} \widetilde{A}_{1} T_{n} v_{n}\right)\right\| \\
\leq & \left\|T_{n+N} v_{n+N}-T_{n} v_{n}\right\|+\left|\rho_{n+N}-\rho_{n}\right|\left\|\widetilde{A}_{1} T_{n} v_{n}\right\| \\
\leq & \widetilde{M}_{0} \sum_{k=1}^{M}\left|\lambda_{k, n+N}-\lambda_{k, n}\right|+\left\|u_{n+N}-u_{n}\right\| \\
& +\frac{4 \widetilde{M}_{1}\left|s_{n+N}-s_{n}\right|+\left|\rho_{n+N}-\rho_{n}\right|\left\|\widetilde{A}_{1} T_{n} v_{n}\right\|}{L} \\
\leq & \widetilde{M}_{0} \sum_{k=1}^{M}\left|\lambda_{k, n+N}-\lambda_{k, n}\right|+\widetilde{M}_{2} \sum_{j=1}^{K}\left|r_{j, n+N}-r_{j, n}\right| \\
& +\left\|x_{n+N}-x_{n}\right\|+\frac{4 \widetilde{M}_{1}}{L}\left|s_{n+N}-s_{n}\right|+\left|\rho_{n+N}-\rho_{n}\right|\left\|\widetilde{A}_{1} T_{n} v_{n}\right\| .
\end{aligned}
$$

From Lemma 11 and (64), it is found that

$$
\begin{aligned}
& \left\|x_{n+N}-x_{n}\right\| \\
& =\| y_{n+N-1}-\mu \alpha_{n+N-1} \widetilde{A}_{2} y_{n+N-1} \\
& \quad-\left(y_{n-1}-\mu \alpha_{n-1} \widetilde{A}_{2} y_{n-1}\right) \| \\
& =\left\|S_{[n+N]}^{\alpha_{n+N-1}} z_{n+N-1}-S_{[n]}^{\alpha_{n-1}} z_{n-1}\right\| \\
& \leq\left\|S_{[n+N]}^{\alpha_{n+N-1}} z_{n+N-1}-S_{[n+N]}^{\alpha_{n+N-1}} z_{n-1}\right\| \\
& \quad+\left\|S_{[n+N]}^{\alpha_{n+N-1}} z_{n-1}-S_{[n]}^{\alpha_{n-1}} z_{n-1}\right\|
\end{aligned}
$$

$$
\begin{aligned}
& \leq\left(1-\alpha_{n+N-1} \tau\right)\left\|z_{n+N-1}-z_{n-1}\right\| \\
& +\mu\left|\alpha_{n+N-1}-\alpha_{n-1}\right|\left\|\widetilde{A}_{2} S_{[n]} z_{n-1}\right\| \\
& =\left(1-\alpha_{n+N-1} \tau\right)\left\|z_{n+N-1}-z_{n-1}\right\| \\
& +\mu\left|\alpha_{n+N-1}-\alpha_{n-1}\right|\left\|\widetilde{A}_{2} y_{n-1}\right\| \\
& \leq\left(1-\alpha_{n+N-1} \tau\right) \\
& \times\left[\widetilde{M}_{0} \sum_{k=1}^{M}\left|\lambda_{k, n+N-1}-\lambda_{k, n-1}\right|\right. \\
& +\widetilde{M}_{2} \sum_{j=1}^{K}\left|r_{j, n+N-1}-r_{j, n-1}\right|+\left\|x_{n+N-1}-x_{n-1}\right\| \\
& +\frac{4 \widetilde{M}_{1}}{L}\left|s_{n+N-1}-s_{n-1}\right| \\
& \left.+\left|\rho_{n+N-1}-\rho_{n-1}\right|\left\|\widetilde{A}_{1} T_{n-1} v_{n-1}\right\|\right] \\
& +\mu\left|\alpha_{n+N-1}-\alpha_{n-1}\right|\left\|\widetilde{A}_{2} y_{n-1}\right\| \\
& \leq\left(1-\alpha_{n+N-1} \tau\right)\left\|x_{n+N-1}-x_{n-1}\right\| \\
& +\widetilde{M}_{0} \sum_{k=1}^{M}\left|\lambda_{k, n+N-1}-\lambda_{k, n-1}\right|+\widetilde{M}_{2} \sum_{j=1}^{K}\left|r_{j, n+N-1}-r_{j, n-1}\right| \\
& +\frac{4 \widetilde{M}_{1}}{L}\left|s_{n+N-1}-s_{n-1}\right|+\left|\rho_{n+N-1}-\rho_{n-1}\right|\left\|\widetilde{A}_{1} T_{n-1} v_{n-1}\right\| \\
& +\mu\left|\alpha_{n+N-1}-\alpha_{n-1}\right|\left\|\widetilde{A}_{2} y_{n-1}\right\| \\
& \leq\left(1-\alpha_{n+N-1} \tau\right)\left\|x_{n+N-1}-x_{n-1}\right\| \\
& +\widetilde{M}_{3}\left(\sum_{k=1}^{M}\left|\lambda_{k, n+N-1}-\lambda_{k, n-1}\right|+\sum_{j=1}^{K}\left|r_{j, n+N-1}-r_{j, n-1}\right|\right. \\
& +\left|s_{n+N-1}-s_{n-1}\right|+\left|\rho_{n+N-1}-\rho_{n-1}\right| \\
& \left.+\left|\alpha_{n+N-1}-\alpha_{n-1}\right|\right)
\end{aligned}
$$

where $\sup _{n \geq 0}\left\{\widetilde{M}_{0}+4 \widetilde{M}_{1} / L+\widetilde{M}_{2}+\left\|\widetilde{A}_{1} T_{n} v_{n}\right\|+\mu\left\|\widetilde{A}_{2} y_{n}\right\|\right\} \leq \widetilde{M}_{3}$ for some $\widetilde{M}_{3}>0$. Applying Lemma 12 to (65) we obtain from conditions (i)-(vi) that

$$
\lim _{n \rightarrow \infty}\left\|x_{n+N}-x_{n}\right\|=0
$$

Step 3. We prove that $\lim _{n \rightarrow \infty}\left\|x_{n}-S_{[n+N]} \cdots S_{[n+1]} x_{n}\right\|=0$ provided $\lim _{n \rightarrow \infty}\left(\left\|x_{n}-y_{n}\right\|+\left\|T_{n} v_{n}-v_{n}\right\|\right)=0$.

Indeed, from $\left\|x_{n+1}-y_{n}\right\|=\mu \alpha_{n}\left\|\widetilde{A}_{2} y_{n}\right\| \leq \alpha_{n} \widetilde{M}_{3}$ and condition (i), we get $\lim _{n \rightarrow \infty}\left\|x_{n+1}-y_{n}\right\|=0$. Now, let us show that $\left\|u_{n}-x_{n}\right\| \rightarrow 0,\left\|v_{n}-u_{n}\right\| \rightarrow 0$ and $\left\|x_{n}-T_{n} v_{n}\right\| \rightarrow 0$ 
as $n \rightarrow \infty$. As a matter of fact, utilizing Lemma 4, we get from (43)

$$
\begin{aligned}
\left\|y_{n}-x^{*}\right\|^{2} & =\left\|S_{[n+1]}\left(T_{n} v_{n}-\rho_{n} \widetilde{A}_{1} T_{n} v_{n}\right)-x^{*}\right\|^{2} \\
& \leq\left\|T_{n} v_{n}-x^{*}-\rho_{n} \widetilde{A}_{1} T_{n} v_{n}\right\|^{2} \\
& \leq\left\|T_{n} v_{n}-x^{*}\right\|^{2}-2 \rho_{n}\left\langle\widetilde{A}_{1} T_{n} v_{n}, z_{n}-x^{*}\right\rangle \\
& \leq\left\|v_{n}-x^{*}\right\|^{2}+2 \rho_{n}\left\|\widetilde{A}_{1} T_{n} v_{n}\right\|\left\|z_{n}-x^{*}\right\| .
\end{aligned}
$$

Observe that

$$
\begin{aligned}
& \left\|\Delta_{n}^{j} x_{n}-x^{*}\right\|^{2} \\
& =\left\|T_{r_{j, n}}^{\left(\Theta_{j}, \varphi_{j}\right)}\left(I-r_{j, n} A_{j}\right) \Delta_{n}^{j-1} x_{n}-T_{r_{j, n}}^{\left(\Theta_{j}, \varphi_{j}\right)}\left(I-r_{j, n} A_{j}\right) x^{*}\right\|^{2} \\
& \leq\left\|\left(I-r_{j, n} A_{j}\right) \Delta_{n}^{j-1} x_{n}-\left(I-r_{j, n} A_{j}\right) x^{*}\right\|^{2} \\
& \leq\left\|\Delta_{n}^{j-1} x_{n}-x^{*}\right\|^{2}+r_{j, n}\left(r_{j, n}-2 \zeta_{j}\right)\left\|A_{j} \Delta_{n}^{j-1} x_{n}-A_{j} x^{*}\right\|^{2} \\
& \leq\left\|x_{n}-x^{*}\right\|^{2}+r_{j, n}\left(r_{j, n}-2 \zeta_{j}\right)\left\|A_{j} \Delta_{n}^{j-1} x_{n}-A_{j} x^{*}\right\|^{2} \\
& \left\|\Lambda_{n}^{k} u_{n}-x^{*}\right\|^{2} \\
& =\left\|J_{R_{k}, \lambda_{k, n}}\left(I-\lambda_{k, n} B_{k}\right) \Lambda_{n}^{k-1} u_{n}-J_{R_{k}, \lambda_{k, n}}\left(I-\lambda_{k, n} B_{k}\right) x^{*}\right\|^{2} \\
& \leq\left\|\left(I-\lambda_{k, n} B_{k}\right) \Lambda_{n}^{k-1} u_{n}-\left(I-\lambda_{k, n} B_{k}\right) x^{*}\right\|^{2} \\
& \leq\left\|\Lambda_{n}^{k-1} u_{n}-x^{*}\right\|^{2}+\lambda_{k, n}\left(\lambda_{k, n}-2 \eta_{k}\right)\left\|B_{k} \Lambda_{n}^{k-1} u_{n}-B_{k} x^{*}\right\|^{2} \\
& \leq\left\|u_{n}-x^{*}\right\|^{2}+\lambda_{k, n}\left(\lambda_{k, n}-2 \eta_{k}\right)\left\|B_{k} \Lambda_{n}^{k-1} u_{n}-B_{k} x^{*}\right\|^{2} \\
& \leq\left\|x_{n}-x^{*}\right\|^{2}+\lambda_{k, n}\left(\lambda_{k, n}-2 \eta_{k}\right)\left\|B_{k} \Lambda_{n}^{k-1} u_{n}-B_{k} x^{*}\right\|^{2}
\end{aligned}
$$

for $j \in\{1,2, \ldots, K\}$ and $k \in\{1,2, \ldots, M\}$. Combining (67)(68), we get

$$
\begin{aligned}
& \left\|y_{n}-x^{*}\right\|^{2} \\
& \leq\left\|v_{n}-x^{*}\right\|^{2}+2 \rho_{n}\left\|\widetilde{A}_{1} T_{n} v_{n}\right\|\left\|z_{n}-x^{*}\right\| \\
& \leq\left\|\Lambda_{n}^{k} u_{n}-x^{*}\right\|^{2}+2 \rho_{n}\left\|\widetilde{A}_{1} T_{n} v_{n}\right\|\left\|z_{n}-x^{*}\right\| \\
& \leq\left\|u_{n}-x^{*}\right\|^{2}+\lambda_{k, n}\left(\lambda_{k, n}-2 \eta_{k}\right)\left\|B_{k} \Lambda_{n}^{k-1} u_{n}-B_{k} x^{*}\right\|^{2} \\
& \quad+2 \rho_{n}\left\|\widetilde{A}_{1} T_{n} v_{n}\right\|\left\|z_{n}-x^{*}\right\| \\
& \leq\left\|\Delta_{n}^{j} x_{n}-x^{*}\right\|^{2}+\lambda_{k, n}\left(\lambda_{k, n}-2 \eta_{k}\right)\left\|B_{k} \Lambda_{n}^{k-1} u_{n}-B_{k} x^{*}\right\|^{2} \\
& \quad+2 \rho_{n}\left\|\widetilde{A}_{1} T_{n} v_{n}\right\|\left\|z_{n}-x^{*}\right\|
\end{aligned}
$$

$$
\begin{aligned}
\leq & \left\|x_{n}-x^{*}\right\|^{2}+r_{j, n}\left(r_{j, n}-2 \zeta_{j}\right)\left\|A_{j} \Delta_{n}^{j-1} x_{n}-A_{j} x^{*}\right\|^{2} \\
& +\lambda_{k, n}\left(\lambda_{k, n}-2 \eta_{k}\right)\left\|B_{k} \Lambda_{n}^{k-1} u_{n}-B_{k} x^{*}\right\|^{2} \\
& +2 \rho_{n}\left\|\widetilde{A}_{1} T_{n} v_{n}\right\|\left\|z_{n}-x^{*}\right\|,
\end{aligned}
$$

which immediately yields

$$
\begin{aligned}
& r_{j, n}\left(2 \zeta_{j}-r_{j, n}\right)\left\|A_{j} \Delta_{n}^{j-1} x_{n}-A_{j} x^{*}\right\|^{2} \\
& \quad+\lambda_{k, n}\left(2 \eta_{k}-\lambda_{k, n}\right)\left\|B_{k} \Lambda_{n}^{k-1} u_{n}-B_{k} x^{*}\right\|^{2} \\
& \leq\left\|x_{n}-x^{*}\right\|^{2}-\left\|y_{n}-x^{*}\right\|^{2}+2 \rho_{n}\left\|\widetilde{A}_{1} T_{n} v_{n}\right\|\left\|z_{n}-x^{*}\right\| \\
& \leq\left\|x_{n}-y_{n}\right\|\left(\left\|x_{n}-x^{*}\right\|+\left\|y_{n}-x^{*}\right\|\right) \\
& \quad+2 \rho_{n}\left\|\widetilde{A}_{1} T_{n} v_{n}\right\|\left\|z_{n}-x^{*}\right\| .
\end{aligned}
$$

Since $\left\{\lambda_{k, n}\right\}_{n=0}^{\infty} \subset\left[a_{k}, b_{k}\right] \subset\left(0,2 \eta_{k}\right)$ and $\left\{r_{j, n}\right\}_{n=0}^{\infty} \subset$ $\left[c_{j}, d_{j}\right] \subset\left(0,2 \zeta_{j}\right)$ for $j=1,2, \ldots, K$ and $k=1,2, \ldots, M$ and $\left\{x_{n}\right\},\left\{y_{n}\right\},\left\{\widetilde{A}_{1} T_{n} v_{n}\right\}$ and $\left\{z_{n}\right\}$ are bounded sequences, we deduce from $\rho_{n} \rightarrow 0$ and $\left\|x_{n}-y_{n}\right\| \rightarrow 0$ that

$$
\begin{aligned}
& \lim _{n \rightarrow \infty}\left\|A_{j} \Delta_{n}^{j-1} x_{n}-A_{j} x^{*}\right\|=0, \\
& \lim _{n \rightarrow \infty}\left\|B_{k} \Lambda_{n}^{k-1} u_{n}-B_{k} x^{*}\right\|=0,
\end{aligned}
$$

for all $j \in\{1,2, \ldots, K\}$ and $k \in\{1,2, \ldots, M\}$.

Furthermore, by Proposition 3(ii) and Lemma 5(a), we have

$\left\|\Delta_{n}^{j} x_{n}-x^{*}\right\|^{2}$

$$
\begin{aligned}
& =\left\|T_{r_{j, n}}^{\left(\Theta_{j}, \varphi_{j}\right)}\left(I-r_{j, n} A_{j}\right) \Delta_{n}^{j-1} x_{n}-T_{r_{j, n}}^{\left(\Theta_{j}, \varphi_{j}\right)}\left(I-r_{j, n} A_{j}\right) x^{*}\right\|^{2} \\
& \leq\left\langle\left(I-r_{j, n} A_{j}\right) \Delta_{n}^{j-1} x_{n}-\left(I-r_{j, n} A_{j}\right) x^{*}, \Delta_{n}^{j} x_{n}-x^{*}\right\rangle \\
& =\frac{1}{2} \\
& \times\left(\left\|\left(I-r_{j, n} A_{j}\right) \Delta_{n}^{j-1} x_{n}-\left(I-r_{j, n} A_{j}\right) x^{*}\right\|^{2}\right. \\
& \quad+\left\|\Delta_{n}^{j} x_{n}-x^{*}\right\|^{2} \\
& \left.\quad-\left\|\left(I-r_{j, n} A_{j}\right) \Delta_{n}^{j-1} x_{n}-\left(I-r_{j, n} A_{j}\right) x^{*}-\left(\Delta_{n}^{j} x_{n}-x^{*}\right)\right\|^{2}\right) \\
& \leq \frac{1}{2}\left(\left\|\Delta_{n}^{j-1} x_{n}-x^{*}\right\|^{2}+\left\|\Delta_{n}^{j} x_{n}-x^{*}\right\|^{2}\right. \\
& \left.\quad-\left\|\Delta_{n}^{j-1} x_{n}-\Delta_{n}^{j} x_{n}-r_{j, n}\left(A_{j} \Delta_{n}^{j-1} x_{n}-A_{j} x^{*}\right)\right\|^{2}\right),
\end{aligned}
$$


which implies that

$$
\begin{aligned}
&\left\|\Delta_{n}^{j} x_{n}-x^{*}\right\|^{2} \\
& \leq\left\|\Delta_{n}^{j-1} x_{n}-x^{*}\right\|^{2} \\
& \quad-\left\|\Delta_{n}^{j-1} x_{n}-\Delta_{n}^{j} x_{n}-r_{j, n}\left(A_{j} \Delta_{n}^{j-1} x_{n}-A_{j} x^{*}\right)\right\|^{2} \\
&=\left\|\Delta_{n}^{j-1} x_{n}-x^{*}\right\|^{2}-\left\|\Delta_{n}^{j-1} x_{n}-\Delta_{n}^{j} x_{n}\right\|^{2} \\
& \quad-r_{j, n}^{2}\left\|A_{j} \Delta_{n}^{j-1} x_{n}-A_{j} x^{*}\right\|^{2} \\
& \quad+2 r_{j, n}\left\langle\Delta_{n}^{j-1} x_{n}-\Delta_{n}^{j} x_{n}, A_{j} \Delta_{n}^{j-1} x_{n}-A_{j} x^{*}\right\rangle \\
& \leq\left\|\Delta_{n}^{j-1} x_{n}-x^{*}\right\|^{2}-\left\|\Delta_{n}^{j-1} x_{n}-\Delta_{n}^{j} x_{n}\right\|^{2} \\
&+2 r_{j, n}\left\|\Delta_{n}^{j-1} x_{n}-\Delta_{n}^{j} x_{n}\right\|\left\|A_{j} \Delta_{n}^{j-1} x_{n}-A_{j} x^{*}\right\| \\
& \leq\left\|x_{n}-x^{*}\right\|^{2}-\left\|\Delta_{n}^{j-1} x_{n}-\Delta_{n}^{j} x_{n}\right\|^{2} \\
&+2 r_{j, n}\left\|\Delta_{n}^{j-1} x_{n}-\Delta_{n}^{j} x_{n}\right\|\left\|A_{j} \Delta_{n}^{j-1} x_{n}-A_{j} x^{*}\right\| .
\end{aligned}
$$

By Lemma 5(a) and Lemma 14, we obtain

$$
\begin{aligned}
& \left\|\Lambda_{n}^{k} u_{n}-x^{*}\right\|^{2} \\
& =\left\|J_{R_{k}, \lambda_{k, n}}\left(I-\lambda_{k, n} B_{k}\right) \Lambda_{n}^{k-1} u_{n}-J_{R_{k}, \lambda_{k, n}}\left(I-\lambda_{k, n} B_{k}\right) x^{*}\right\|^{2} \\
& \leq\left\langle\left(I-\lambda_{k, n} B_{k}\right) \Lambda_{n}^{k-1} u_{n}-\left(I-\lambda_{k, n} B_{k}\right) x^{*}, \Lambda_{n}^{k} u_{n}-x^{*}\right\rangle \\
& =\frac{1}{2}\left(\left\|\left(I-\lambda_{k, n} B_{k}\right) \Lambda_{n}^{k-1} u_{n}-\left(I-\lambda_{k, n} B_{k}\right) x^{*}\right\|^{2}\right. \\
& \quad+\left\|\Lambda_{n}^{k} u_{n}-x^{*}\right\|^{2} \\
& \quad-\|\left(I-\lambda_{k, n} B_{k}\right) \Lambda_{n}^{k-1} u_{n} \\
& \left.\quad-\left(I-\lambda_{k, n} B_{k}\right) x^{*}-\left(\Lambda_{n}^{k} u_{n}-x^{*}\right) \|^{2}\right) \\
& \leq \frac{1}{2}\left(\left\|\Lambda_{n}^{k-1} u_{n}-x^{*}\right\|^{2}+\left\|\Lambda_{n}^{k} u_{n}-x^{*}\right\|^{2}\right. \\
& \left.\quad-\left\|\Lambda_{n}^{k-1} u_{n}-\Lambda_{n}^{k} u_{n}-\lambda_{k, n}\left(B_{k} \Lambda_{n}^{k-1} u_{n}-B_{k} x^{*}\right)\right\|^{2}\right) \\
& \leq \frac{1}{2}\left(\left\|u_{n}-x^{*}\right\|^{2}+\left\|\Lambda_{n}^{k} u_{n}-x^{*}\right\|^{2}\right. \\
& \left.\quad-\left\|\Lambda_{n}^{k-1} u_{n}-\Lambda_{n}^{k} u_{n}-\lambda_{k, n}\left(B_{k} \Lambda_{n}^{k-1} u_{n}-B_{k} x^{*}\right)\right\|^{2}\right) \\
& \left.\quad-\left\|\Lambda_{n}^{k-1} u_{n}-\Lambda_{n}^{k} u_{n}-\lambda_{k, n}\left(B_{k} \Lambda_{n}^{k-1} u_{n}-B_{k} x^{*}\right)\right\|^{2}\right), \\
& \leq \frac{1}{2}\left(\left\|x_{n}-x^{*}\right\|^{2}+\left\|\Lambda_{n}^{k} u_{n}-x^{*}\right\|^{2}\right.
\end{aligned}
$$

which immediately leads to

$$
\begin{aligned}
& \left\|\Lambda_{n}^{k} u_{n}-x^{*}\right\|^{2} \\
& \leq\left\|x_{n}-x^{*}\right\|^{2} \\
& \quad-\left\|\Lambda_{n}^{k-1} u_{n}-\Lambda_{n}^{k} u_{n}-\lambda_{k, n}\left(B_{k} \Lambda_{n}^{k-1} u_{n}-B_{k} x^{*}\right)\right\|^{2} \\
& =\left\|x_{n}-x^{*}\right\|^{2}-\left\|\Lambda_{n}^{k-1} u_{n}-\Lambda_{n}^{k} u_{n}\right\|^{2} \\
& \quad-\lambda_{k, n}^{2}\left\|B_{k} \Lambda_{n}^{k-1} u_{n}-B_{k} x^{*}\right\|^{2} \\
& \quad+2 \lambda_{k, n}\left\langle\Lambda_{n}^{k-1} u_{n}-\Lambda_{n}^{k} u_{n}, B_{k} \Lambda_{n}^{k-1} u_{n}-B_{k} x^{*}\right\rangle \\
& \leq \\
& \quad\left\|x_{n}-x^{*}\right\|^{2}-\left\|\Lambda_{n}^{k-1} u_{n}-\Lambda_{n}^{k} u_{n}\right\|^{2} \\
& \quad+2 \lambda_{k, n}\left\|\Lambda_{n}^{k-1} u_{n}-\Lambda_{n}^{k} u_{n}\right\|\left\|B_{k} \Lambda_{n}^{k-1} u_{n}-B_{k} x^{*}\right\| .
\end{aligned}
$$

Combining (67) and (75) we conclude that

$$
\begin{aligned}
\left\|y_{n}-x^{*}\right\|^{2} \leq & \left\|v_{n}-x^{*}\right\|^{2}+2 \rho_{n}\left\|\widetilde{A}_{1} T_{n} v_{n}\right\|\left\|z_{n}-x^{*}\right\| \\
\leq & \left\|\Lambda_{n}^{k} u_{n}-x^{*}\right\|^{2}+2 \rho_{n}\left\|\widetilde{A}_{1} T_{n} v_{n}\right\|\left\|z_{n}-x^{*}\right\| \\
\leq & \left\|x_{n}-x^{*}\right\|^{2}-\left\|\Lambda_{n}^{k-1} u_{n}-\Lambda_{n}^{k} u_{n}\right\|^{2} \\
& +2 \lambda_{k, n}\left\|\Lambda_{n}^{k-1} u_{n}-\Lambda_{n}^{k} u_{n}\right\|\left\|B_{k} \Lambda_{n}^{k-1} u_{n}-B_{k} x^{*}\right\| \\
& +2 \rho_{n}\left\|\widetilde{A}_{1} T_{n} v_{n}\right\|\left\|z_{n}-x^{*}\right\|,
\end{aligned}
$$

which yields

$$
\begin{aligned}
& \left\|\Lambda_{n}^{k-1} u_{n}-\Lambda_{n}^{k} u_{n}\right\|^{2} \\
& \leq \quad\left\|x_{n}-x^{*}\right\|^{2}-\left\|y_{n}-x^{*}\right\|^{2} \\
& \quad+2 \lambda_{k, n}\left\|\Lambda_{n}^{k-1} u_{n}-\Lambda_{n}^{k} u_{n}\right\|\left\|B_{k} \Lambda_{n}^{k-1} u_{n}-B_{k} x^{*}\right\| \\
& \quad+2 \rho_{n}\left\|\widetilde{A}_{1} T_{n} v_{n}\right\|\left\|z_{n}-x^{*}\right\| \\
& \leq \quad\left\|x_{n}-y_{n}\right\|\left(\left\|x_{n}-x^{*}\right\|+\left\|y_{n}-x^{*}\right\|\right) \\
& \quad+2 \lambda_{k, n}\left\|\Lambda_{n}^{k-1} u_{n}-\Lambda_{n}^{k} u_{n}\right\|\left\|B_{k} \Lambda_{n}^{k-1} u_{n}-B_{k} x^{*}\right\| \\
& \quad+2 \rho_{n}\left\|\widetilde{A}_{1} T_{n} v_{n}\right\|\left\|z_{n}-x^{*}\right\| .
\end{aligned}
$$

Since $\left\{\lambda_{k, n}\right\}_{n=0}^{\infty} \subset\left[a_{k}, b_{k}\right] \subset\left(0,2 \eta_{k}\right)$ for $k=1,2, \ldots, M$ and $\left\{u_{n}\right\},\left\{x_{n}\right\},\left\{y_{n}\right\},\left\{\widetilde{A}_{1} T_{n} v_{n}\right\}$ and $\left\{z_{n}\right\}$ are bounded sequences, we deduce from (71), $\rho_{n} \rightarrow 0$, and $\left\|x_{n}-y_{n}\right\| \rightarrow 0$ that

$$
\lim _{n \rightarrow \infty}\left\|\Lambda_{n}^{k-1} u_{n}-\Lambda_{n}^{k} u_{n}\right\|=0, \quad \forall k \in\{1,2, \ldots, M\}
$$


Also, combining (51), (67), and (73), we deduce that

$$
\begin{aligned}
\left\|y_{n}-x^{*}\right\|^{2} \leq & \left\|v_{n}-x^{*}\right\|^{2}+2 \rho_{n}\left\|\widetilde{A}_{1} T_{n} v_{n}\right\|\left\|z_{n}-x^{*}\right\| \\
\leq & \left\|u_{n}-x^{*}\right\|^{2}+2 \rho_{n}\left\|\widetilde{A}_{1} T_{n} v_{n}\right\|\left\|z_{n}-x^{*}\right\| \\
\leq & \left\|\Delta_{n}^{j} x_{n}-x^{*}\right\|^{2}+2 \rho_{n}\left\|\widetilde{A}_{1} T_{n} v_{n}\right\|\left\|z_{n}-x^{*}\right\| \\
\leq & \left\|x_{n}-x^{*}\right\|^{2}-\left\|\Delta_{n}^{j-1} x_{n}-\Delta_{n}^{j} x_{n}\right\|^{2} \\
& +2 r_{j, n}\left\|\Delta_{n}^{j-1} x_{n}-\Delta_{n}^{j} x_{n}\right\|\left\|A_{j} \Delta_{n}^{j-1} x_{n}-A_{j} x^{*}\right\| \\
& +2 \rho_{n}\left\|\widetilde{A}_{1} T_{n} v_{n}\right\|\left\|z_{n}-x^{*}\right\|,
\end{aligned}
$$

which leads to

$$
\begin{aligned}
\| \Delta_{n}^{j-1} x_{n} & -\Delta_{n}^{j} x_{n} \|^{2} \\
\leq & \left\|x_{n}-x^{*}\right\|^{2}-\left\|y_{n}-x^{*}\right\|^{2} \\
& +2 r_{j, n}\left\|\Delta_{n}^{j-1} x_{n}-\Delta_{n}^{j} x_{n}\right\|\left\|A_{j} \Delta_{n}^{j-1} x_{n}-A_{j} x^{*}\right\| \\
& +2 \rho_{n}\left\|\widetilde{A}_{1} T_{n} v_{n}\right\|\left\|z_{n}-x^{*}\right\| \\
\leq & \left\|x_{n}-y_{n}\right\|\left(\left\|x_{n}-x^{*}\right\|+\left\|y_{n}-x^{*}\right\|\right) \\
& +2 r_{j, n}\left\|\Delta_{n}^{j-1} x_{n}-\Delta_{n}^{j} x_{n}\right\|\left\|A_{j} \Delta_{n}^{j-1} x_{n}-A_{j} x^{*}\right\| \\
& +2 \rho_{n}\left\|\widetilde{A}_{1} T_{n} v_{n}\right\|\left\|z_{n}-x^{*}\right\| .
\end{aligned}
$$

Since $\left\{r_{j, n}\right\}_{n=0}^{\infty} \subset\left[c_{j}, d_{j}\right] \subset\left(0,2 \zeta_{j}\right)$ for $j=1,2, \ldots, K$ and $\left\{x_{n}\right\},\left\{y_{n}\right\},\left\{\widetilde{A}_{1} T_{n} v_{n}\right\}$ and $\left\{z_{n}\right\}$ are bounded sequences, we conclude from (71), $\rho_{n} \rightarrow 0$, and $\left\|x_{n}-y_{n}\right\| \rightarrow 0$ that

$$
\lim _{n \rightarrow \infty}\left\|\Delta_{n}^{j-1} x_{n}-\Delta_{n}^{j} x_{n}\right\|=0, \quad \forall j \in\{1,2, \ldots, K\} .
$$

Hence from (78) and (81) we get

$$
\begin{aligned}
\left\|x_{n}-u_{n}\right\|= & \left\|\Delta_{n}^{0} x_{n}-\Delta_{n}^{K} x_{n}\right\| \\
\leq & \left\|\Delta_{n}^{0} x_{n}-\Delta_{n}^{1} x_{n}\right\|+\left\|\Delta_{n}^{1} x_{n}-\Delta_{n}^{2} x_{n}\right\|+\cdots \\
& +\left\|\Delta_{n}^{K-1} x_{n}-\Delta_{n}^{K} x_{n}\right\| \longrightarrow 0 \text { as } n \longrightarrow \infty, \\
\left\|u_{n}-v_{n}\right\|= & \left\|\Lambda_{n}^{0} u_{n}-\Lambda_{n}^{M} u_{n}\right\| \\
\leq & \left\|\Lambda_{n}^{0} u_{n}-\Lambda_{n}^{1} u_{n}\right\|+\left\|\Lambda_{n}^{1} u_{n}-\Lambda_{n}^{2} u_{n}\right\|+\cdots \\
& +\left\|\Lambda_{n}^{M-1} u_{n}-\Lambda_{n}^{M} u_{n}\right\| \longrightarrow 0 \text { as } n \longrightarrow \infty,
\end{aligned}
$$

respectively. Thus, from (82) and (83), we obtain

$$
\left\|x_{n}-v_{n}\right\| \leq\left\|x_{n}-u_{n}\right\|+\left\|u_{n}-v_{n}\right\| \longrightarrow 0 \quad \text { as } n \longrightarrow \infty \text {, }
$$

together with $\left\|v_{n}-T_{n} v_{n}\right\| \rightarrow 0$, which implies that

$$
\lim _{n \rightarrow \infty}\left\|x_{n}-T_{n} v_{n}\right\|=0 \text {. }
$$

On the other hand, we observe that the following relation holds:

$$
\begin{aligned}
x_{n+N}-x_{n}= & x_{n+N}-S_{[n+N]}\left(I-\rho_{n+N-1} \widetilde{A}_{1}\right) T_{n+N-1} v_{n+N-1} \\
& +S_{[n+N]}\left(I-\rho_{n+N-1} \widetilde{A}_{1}\right) T_{n+N-1} v_{n+N-1} \\
& -S_{[n+N]} S_{[n+N-1]}\left(I-\rho_{n+N-2} \widetilde{A}_{1}\right) T_{n+N-2} v_{n+N-2} \\
& +\cdots+S_{[n+N]} \cdots S_{[n+2]}\left(I-\rho_{n+1} \widetilde{A}_{1}\right) T_{n+1} v_{n+1} \\
& -S_{[n+N]} \cdots S_{[n+1]}\left(I-\rho_{n} \widetilde{A}_{1}\right) T_{n} v_{n} \\
& +S_{[n+N]} \cdots S_{[n+1]}\left(I-\rho_{n} \widetilde{A}_{1}\right) T_{n} v_{n}-x_{n} .
\end{aligned}
$$

Since $\left\|x_{n+1}-y_{n}\right\| \rightarrow 0$ and $\rho_{n} \rightarrow 0$ as $n \rightarrow \infty$, from the nonexpansivity of each $S_{i}(i=1,2, \ldots, N)$ and boundedness of $\left\{\widetilde{A}_{1} T_{n} v_{n}\right\}$ it follows from (85) that as $n \rightarrow \infty$ we have

$$
\begin{aligned}
& \left\|x_{n+N}-S_{[n+N]}\left(I-\rho_{n+N-1} \widetilde{A}_{1}\right) T_{n+N-1} v_{n+N-1}\right\| \\
& =\left\|x_{n+N}-y_{n+N-1}\right\| \longrightarrow 0, \\
\| S_{[n+N]}\left(I-\rho_{n+N-1} \widetilde{A}_{1}\right) T_{n+N-1} v_{n+N-1} & S_{[n+N]} S_{[n+N-1]}\left(I-\rho_{n+N-2} \widetilde{A}_{1}\right) T_{n+N-2} v_{n+N-2} \| \\
\leq & \|\left(I-\rho_{n+N-1} \widetilde{A}_{1}\right) T_{n+N-1} v_{n+N-1} \\
& -S_{[n+N-1]}\left(I-\rho_{n+N-2} \widetilde{A}_{1}\right) T_{n+N-2} v_{n+N-2} \| \\
\leq & \| T_{n+N-1} v_{n+N-1} \\
& -S_{[n+N-1]}\left(I-\rho_{n+N-2} \widetilde{A_{1}}\right) T_{n+N-2} v_{n+N-2} \| \\
& +\rho_{n+N-1}\left\|\widetilde{A}_{1} T_{n+N-1} v_{n+N-1}\right\| \\
\leq & \left\|T_{n+N-1} v_{n+N-1}-x_{n+N-1}\right\| \\
& +\left\|x_{n+N-1}-S_{[n+N-1]}\left(I-\rho_{n+N-2} \widetilde{A_{1}}\right) T_{n+N-2} v_{n+N-2}\right\| \\
& +\rho_{n+N-1}\left\|\widetilde{A}_{1} T_{n+N-1} v_{n+N-1}\right\| \\
= & \left\|T_{n+N-1} v_{n+N-1}-x_{n+N-1}\right\|+\left\|x_{n+N-1}-y_{n+N-2}\right\| \\
& \rho_{n+N-1}\left\|\widetilde{A}_{1} T_{n+N-1} v_{n+N-1}\right\| \longrightarrow 0,
\end{aligned}
$$$$
\| S_{[n+N]} \cdots S_{[n+2]}\left(I-\rho_{n+1} \widetilde{A}_{1}\right) T_{n+1} v_{n+1}
$$$$
-S_{[n+N]} \cdots S_{[n+1]}\left(I-\rho_{n} \widetilde{A}_{1}\right) T_{n} v_{n} \|
$$$$
\leq\left\|\left(I-\rho_{n+1} \widetilde{A}_{1}\right) T_{n+1} v_{n+1}-S_{[n+1]}\left(I-\rho_{n} \widetilde{A}_{1}\right) T_{n} v_{n}\right\|
$$$$
\leq\left\|T_{n+1} v_{n+1}-S_{[n+1]}\left(I-\rho_{n} \widetilde{A}_{1}\right) T_{n} v_{n}\right\|
$$ 


$$
\begin{aligned}
& +\rho_{n+1}\left\|\widetilde{A}_{1} T_{n+1} v_{n+1}\right\| \\
\leq & \left\|T_{n+1} v_{n+1}-x_{n+1}\right\|+\left\|x_{n+1}-S_{[n+1]}\left(I-\rho_{n} \widetilde{A}_{1}\right) T_{n} v_{n}\right\| \\
& +\rho_{n+1}\left\|\widetilde{A}_{1} T_{n+1} v_{n+1}\right\| \\
= & \left\|T_{n+1} v_{n+1}-x_{n+1}\right\|+\left\|x_{n+1}-y_{n}\right\| \\
& +\rho_{n+1}\left\|\widetilde{A}_{1} T_{n+1} v_{n+1}\right\| \longrightarrow 0 .
\end{aligned}
$$

Therefore, from (66) and (86), we obtain

$$
\lim _{n \rightarrow \infty}\left\|S_{[n+N]} \cdots S_{[n+1]}\left(I-\rho_{n} \widetilde{A}_{1}\right) T_{n} v_{n}-x_{n}\right\|=0 .
$$

So, it follows that

$$
\begin{aligned}
& \left\|S_{[n+N]} \cdots S_{[n+1]}\left(I-\rho_{n} \widetilde{A}_{1}\right) x_{n}-x_{n}\right\| \\
& \leq \| S_{[n+N]} \cdots S_{[n+1]}\left(I-\rho_{n} \widetilde{A}_{1}\right) x_{n} \\
& \quad \quad-S_{[n+N]} \cdots S_{[n+1]}\left(I-\rho_{n} \widetilde{A}_{1}\right) T_{n} v_{n} \| \\
& \quad+\left\|S_{[n+N]} \cdots S_{[n+1]}\left(I-\rho_{n} \widetilde{A}_{1}\right) T_{n} v_{n}-x_{n}\right\| \longrightarrow 0 .
\end{aligned}
$$

Observe that

$$
\begin{aligned}
& \left\|S_{[n+N]} \cdots S_{[n+1]} x_{n}-x_{n}\right\| \\
& \leq\left\|S_{[n+N]} \cdots S_{[n+1]} x_{n}-S_{[n+N]} \cdots S_{[n+1]}\left(x_{n}-\rho_{n} \widetilde{A}_{1} x_{n}\right)\right\| \\
& \quad+\left\|S_{[n+N]} \cdots S_{[n+1]}\left(x_{n}-\rho_{n} \widetilde{A}_{1} x_{n}\right)-x_{n}\right\| \\
& \leq \rho_{n}\left\|\widetilde{A}_{1} x_{n}\right\|+\left\|S_{[n+N]} \cdots S_{[n+1]}\left(x_{n}-\rho_{n} \widetilde{A}_{1} x_{n}\right)-x_{n}\right\| \\
& \longrightarrow 0 \quad(n \longrightarrow \infty) .
\end{aligned}
$$

That is,

$$
\lim _{n \rightarrow \infty}\left\|S_{[n+N]} \cdots S_{[n+1]} x_{n}-x_{n}\right\|=0 .
$$

Step 4. We prove that $\lim \sup _{n \rightarrow \infty}\left\langle\widetilde{A}_{1} x^{*}, x^{*}-x_{n}\right\rangle \leq 0$ provided $\lim _{n \rightarrow \infty}\left(\left\|x_{n}-y_{n}\right\|+\left\|T_{n} v_{n}-v_{n}\right\|\right)=0$.

Indeed, choose a subsequence $\left\{x_{n_{i}}\right\}$ of $\left\{x_{n}\right\}$ such that

$$
\limsup _{n \rightarrow \infty}\left\langle\widetilde{A}_{1} x^{*}, x^{*}-x_{n}\right\rangle=\lim _{i \rightarrow \infty}\left\langle\widetilde{A}_{1} x^{*}, x^{*}-x_{n_{i}}\right\rangle .
$$

The boundedness of $\left\{x_{n_{i}}\right\}$ implies the existence of a subsequence $\left\{x_{n_{i j}}\right\}$ of $\left\{x_{n_{i}}\right\}$ and a point $\hat{x} \in H$ such that $x_{n_{i_{j}}} \rightarrow \widehat{x}$. We may assume without loss of generality that $x_{n_{i}} \rightarrow \widehat{x}$; that is,

$$
\begin{aligned}
\limsup _{n \rightarrow \infty}\left\langle\widetilde{A}_{1} x^{*}, x^{*}-x_{n}\right\rangle & =\lim _{i \rightarrow \infty}\left\langle\widetilde{A}_{1} x^{*}, x^{*}-x_{n_{i}}\right\rangle \\
& =\left\langle\widetilde{A}_{1} x^{*}, x^{*}-\widehat{x}\right\rangle .
\end{aligned}
$$

First, we can readily see that $\widehat{x} \in \cap_{i=1}^{N} \operatorname{Fix}\left(S_{i}\right)$. Since the pool of mappings $\left\{S_{i}: i \leq i \leq N\right\}$ is finite, we may further assume (passing to a further subsequence if necessary) that, for some integer $l \in\{1,2, \ldots, N\}$,

$$
S_{\left[n_{i}\right]} \equiv S_{l}, \quad \forall i \geq 1 .
$$

Then, it follows from (91) that

$$
x_{n_{i}}-S_{[i+N]} \cdots S_{[i+1]} x_{n}-x_{n_{i}} \longrightarrow 0 .
$$

Hence, by Lemma 9, we conclude that

$$
\hat{x} \in \operatorname{Fix}\left(S_{[i+N]} \cdots S_{[i+1]}\right) .
$$

Together with the assumption

$$
\begin{aligned}
\bigcap_{i=1}^{N} \operatorname{Fix}\left(S_{i}\right) & =\operatorname{Fix}\left(S_{1} S_{2} \cdots S_{N}\right) \\
& =\operatorname{Fix}\left(S_{N} S_{1} \cdots S_{N-1}\right) \\
& =\cdots=\operatorname{Fix}\left(S_{2} S_{3} \cdots S_{N} S_{1}\right),
\end{aligned}
$$

this implies that $\widehat{x} \in \cap_{i=1}^{N} \operatorname{Fix}\left(S_{i}\right)$. Now, since

$$
x^{*} \in \mathrm{VI}\left(\bigcap_{i=i}^{N} \operatorname{Fix}\left(S_{i}\right), \widetilde{A}_{1}\right) \text {, }
$$

we obtain

$$
\begin{aligned}
\limsup _{n \rightarrow \infty}\left\langle\widetilde{A}_{1} x^{*}, x^{*}-x_{n}\right\rangle & =\lim _{i \rightarrow \infty}\left\langle\widetilde{A}_{1} x^{*}, x^{*}-x_{n_{i}}\right\rangle \\
& =\left\langle\widetilde{A}_{1} x^{*}, x^{*}-\widehat{x}\right\rangle \leq 0 .
\end{aligned}
$$

Step 5. We prove that $\lim _{n \rightarrow \infty}\left\|x_{n}-x^{*}\right\|=0$ provided $\| x_{n}-$ $y_{n}\|+\| T_{n} v_{n}-v_{n} \|=o\left(\rho_{n}\right)$.

Indeed, first of all, let us show that $\lim \sup _{n \rightarrow \infty}\left\langle\widetilde{A}_{1} x^{*}, x^{*}-x_{n}\right\rangle \leq 0$. We choose a subsequence $\left\{x_{n_{k}}\right\}$ of $\left\{x_{n}\right\}$ such that

$$
\limsup _{n \rightarrow \infty}\left\langle\widetilde{A}_{2} x^{*}, x^{*}-x_{n}\right\rangle=\lim _{k \rightarrow \infty}\left\langle\widetilde{A}_{2} x^{*}, x^{*}-x_{n_{k}}\right\rangle .
$$

The boundedness of $\left\{x_{n_{k}}\right\}$ implies that there is a subsequence of $\left\{x_{n_{k}}\right\}$ which converges weakly to a point $\bar{x} \in H$. Without loss of generality, we may assume that $x_{n_{k}} \rightarrow \bar{x}$; that is,

$$
\begin{aligned}
\limsup _{n \rightarrow \infty}\left\langle\widetilde{A}_{2} x^{*}, x^{*}-x_{n}\right\rangle & =\lim _{k \rightarrow \infty}\left\langle\widetilde{A}_{2} x^{*}, x^{*}-x_{n_{k}}\right\rangle \\
& =\left\langle\widetilde{A}_{2} x^{*}, x^{*}-\bar{x}\right\rangle .
\end{aligned}
$$

Repeating the same argument as in the proof of $\widehat{x} \in \cap_{i=1}^{N} \operatorname{Fix}\left(S_{i}\right)$, we have $\bar{x} \in \cap_{i=1}^{N} \operatorname{Fix}\left(S_{i}\right)$. Let $p \in \cap_{i=1}^{N} \operatorname{Fix}\left(S_{i}\right)$ be fixed arbitrarily. Note that $\cap_{i=i}^{N} \operatorname{Fix}\left(S_{i}\right) \subset \cap_{j=1}^{K} \operatorname{GMEP}\left(\Theta_{j}, \varphi_{j}, A_{j}\right) \cap \cap_{k=1}^{M} I\left(B_{k}, R_{k}\right) \cap \Gamma$. Then, it follows from the nonexpansivity of each 
$S_{i}(i=1,2, \ldots, N)$ and monotonicity of $\widetilde{A}_{1}$ that, for all $n \geq 0$,

$$
\begin{aligned}
\left\|y_{n}-p\right\|^{2}= & \left\|S_{[n+1]}\left(I-\rho_{n} \widetilde{A}_{1}\right) T_{n} v_{n}-S_{[n+1]} p\right\|^{2} \\
\leq & \left\|\left(T_{n} v_{n}-p\right)-\rho_{n} \widetilde{A}_{1} T_{n} v_{n}\right\|^{2} \\
= & \left\|T_{n} v_{n}-p\right\|^{2}+2 \rho_{n}\left\langle\widetilde{A}_{1} T_{n} v_{n}, p-T_{n} v_{n}\right\rangle \\
& +\rho_{n}^{2}\left\|\widetilde{A}_{1} T_{n} v_{n}\right\|^{2} \\
= & \left\|T_{n} v_{n}-p\right\|^{2}+2 \rho_{n}\left\langle\widetilde{A}_{1} T_{n} v_{n}-\widetilde{A}_{1} p, p-T_{n} v_{n}\right\rangle \\
& +2 \rho_{n}\left\langle\widetilde{A}_{1} p, p-T_{n} v_{n}\right\rangle+\rho_{n}^{2}\left\|\widetilde{A}_{1} T_{n} v_{n}\right\|^{2} \\
\leq & \left\|v_{n}-p\right\|^{2}+2 \rho_{n}\left\langle\widetilde{A}_{1} p, p-T_{n} v_{n}\right\rangle \\
& +\rho_{n}^{2}\left\|\widetilde{A}_{1} T_{n} v_{n}\right\|^{2} \\
\leq & \left\|x_{n}-p\right\|^{2}+2 \rho_{n}\left\langle\widetilde{A}_{1} p, p-T_{n} v_{n}\right\rangle+\rho_{n}^{2} \widetilde{M}_{3}^{2},
\end{aligned}
$$

which implies that

$$
\begin{aligned}
\lim _{n \rightarrow \infty} & \left\langle\widetilde{A}_{1} p, p-T_{n} v_{n}\right\rangle \\
& \leq \lim _{n \rightarrow \infty} \frac{1}{2 \rho_{n}}\left[\left\|x_{n}-p\right\|^{2}-\left\|y_{n}-p\right\|^{2}+\rho_{n}^{2} \widetilde{M}_{3}^{2}\right] \\
& \leq \lim _{n \rightarrow \infty} \frac{\left\|x_{n}-y_{n}\right\|}{2 \rho_{n}}\left(\left\|x_{n}-p\right\|+\left\|y_{n}-p\right\|\right)+\lim _{n \rightarrow \infty} \frac{\rho_{n}}{2} \widetilde{M}_{3}^{2} .
\end{aligned}
$$

So, from $\left\|x_{n}-y_{n}\right\|=o\left(\rho_{n}\right)$ and the boundedness of $\left\{x_{n}\right\}$ and $\left\{y_{n}\right\}$, we get

$$
\limsup _{n \rightarrow \infty}\left\langle\widetilde{A}_{1} p, p-T_{n} v_{n}\right\rangle \leq 0,
$$

together with (85), which implies that

$$
\begin{aligned}
& \left\langle\widetilde{A}_{1} p, p-\bar{x}\right\rangle \\
& \quad=\lim _{k \rightarrow \infty}\left\langle\widetilde{A}_{1} p, p-x_{n_{k}}\right\rangle \\
& \quad \leq \limsup _{n \rightarrow \infty}\left\langle\widetilde{A}_{1} p, p-x_{n}\right\rangle \\
& \quad \leq \limsup _{n \rightarrow \infty}\left(\left\langle\widetilde{A}_{1} p, p-T_{n} v_{n}\right\rangle+\left\langle\widetilde{A}_{1} p, T_{n} v_{n}-x_{n}\right\rangle\right) \\
& \quad \leq \limsup _{n \rightarrow \infty}\left\langle\widetilde{A}_{1} p, p-T_{n} v_{n}\right\rangle \\
& \leq 0 .
\end{aligned}
$$

Thus, we have

$$
\left\langle\widetilde{A}_{1} p, p-\bar{x}\right\rangle \leq 0, \quad \forall p \in \bigcap_{i=1}^{N} \operatorname{Fix}\left(S_{i}\right) .
$$

Since $\widetilde{A_{1}}$ is monotone and $1 / \alpha$-Lipschitz continuous, in terms of Minty's lemma [12], we deduce that $\bar{x} \in \operatorname{VI}\left(\cap_{i=1}^{N} \operatorname{Fix}\left(S_{i}\right), \widetilde{A}_{1}\right)$. Therefore, from $\left\{x^{*}\right\}=$ $\operatorname{VI}\left(\operatorname{VI}\left(\Omega, \widetilde{A}_{1}\right), \widetilde{A}_{2}\right)$, we have

$$
\begin{aligned}
\limsup _{n \rightarrow \infty}\left\langle\widetilde{A}_{2} x^{*}, x^{*}-x_{n}\right\rangle & =\lim _{k \rightarrow \infty}\left\langle\widetilde{A}_{2} x^{*}, x^{*}-x_{n_{k}}\right\rangle \\
& =\left\langle\widetilde{A}_{2} x^{*}, x^{*}-\bar{x}\right\rangle \leq 0 .
\end{aligned}
$$

Finally, let us show that $\left\|x_{n}-x^{*}\right\| \rightarrow 0$ as $n \rightarrow \infty$. By utilizing Lemma 11, we deduce from (52) and $S_{[n+1]}^{\alpha_{n}} x^{*}=$ $x^{*}-\alpha_{n} \mu \widetilde{A_{2}} x^{*}$ that for all $n \geq 0$

$\left\|x_{n+1}-x^{*}\right\|$

$=\left\|S_{[n+1]}^{\alpha_{n}} z_{n}-x^{*}\right\|^{2}$

$=\left\|S_{[n+1]}^{\alpha_{n}} z_{n}-S_{[n+1]}^{\alpha_{n}} x^{*}+S_{[n+1]}^{\alpha_{n}} x^{*}-x^{*}\right\|^{2}$

$\leq\left\|S_{[n+1]}^{\alpha_{n}} z_{n}-S_{[n+1]}^{\alpha_{n}} x^{*}\right\|^{2}+2\left\langle S_{[n+1]}^{\alpha_{n}} x^{*}-x^{*}, x_{n+1}-x^{*}\right\rangle$

$\leq\left(1-\alpha_{n} \tau\right)\left\|z_{n}-x^{*}\right\|^{2}-2 \alpha_{n} \mu\left\langle\widetilde{A}_{2} x^{*}, x_{n+1}-x^{*}\right\rangle$

$=\left(1-\alpha_{n} \tau\right)\left\|T_{n} v_{n}-x^{*}-\rho_{n} \widetilde{A}_{1} T_{n} v_{n}\right\|^{2}$

$-2 \alpha_{n} \mu\left\langle\widetilde{A}_{2} x^{*}, x_{n+1}-x^{*}\right\rangle$

$=\left(1-\alpha_{n} \tau\right)\left[\left\|T_{n} v_{n}-x^{*}\right\|^{2}\right.$

$\left.+2 \rho_{n}\left\langle\widetilde{A}_{1} T_{n} v_{n}, x^{*}-T_{n} v_{n}\right\rangle+\rho_{n}^{2}\left\|\widetilde{A}_{1} T_{n} v_{n}\right\|^{2}\right]$

$-2 \alpha_{n} \mu\left\langle\widetilde{A}_{2} x^{*}, x_{n+1}-x^{*}\right\rangle$

$=\left(1-\alpha_{n} \tau\right)\left[\left\|T_{n} v_{n}-x^{*}\right\|^{2}\right.$

$+2 \rho_{n}\left\langle\widetilde{A}_{1} T_{n} v_{n}-\widetilde{A}_{1} x^{*}, x^{*}-T_{n} v_{n}\right\rangle$

$\left.+2 \rho_{n}\left\langle\widetilde{A}_{1} x^{*}, x^{*}-T_{n} v_{n}\right\rangle+\rho_{n}^{2}\left\|\widetilde{A}_{1} T_{n} v_{n}\right\|^{2}\right]$

$-2 \alpha_{n} \mu\left\langle\widetilde{A}_{2} x^{*}, x_{n+1}-x^{*}\right\rangle$

$\leq\left(1-\alpha_{n} \tau\right)\left[\left\|v_{n}-x^{*}\right\|^{2}+2 \rho_{n}\left\langle\widetilde{A}_{1} x^{*}, x^{*}-T_{n} v_{n}\right\rangle\right.$

$\left.+\rho_{n}^{2}\left\|\widetilde{A}_{1} T_{n} v_{n}\right\|^{2}\right]$

$-2 \alpha_{n} \mu\left\langle\widetilde{A}_{2} x^{*}, x_{n+1}-x^{*}\right\rangle$

$\leq\left(1-\alpha_{n} \tau\right)\left[\left\|x_{n}-x^{*}\right\|^{2}+2 \rho_{n}\left\langle\widetilde{A}_{1} x^{*}, x^{*}-T_{n} v_{n}\right\rangle+\rho_{n}^{2} \widetilde{M}_{3}^{2}\right]$

$-2 \alpha_{n} \mu\left\langle\widetilde{A}_{2} x^{*}, x_{n+1}-x^{*}\right\rangle$

$\leq\left(1-\alpha_{n} \tau\right)\left\|x_{n}-x^{*}\right\|^{2}$

$+2 \rho_{n}\left(1-\alpha_{n} \tau\right)\left\langle\widetilde{A}_{1} x^{*}, x^{*}-T_{n} v_{n}\right\rangle+\rho_{n}^{2} \widetilde{M}_{3}^{2}$

$-2 \alpha_{n} \mu\left\langle\widetilde{A}_{2} x^{*}, x_{n+1}-x^{*}\right\rangle$ 


$$
\begin{aligned}
=\left(1-\alpha_{n} \tau\right)\left\|x_{n}-x^{*}\right\|^{2} \\
+\alpha_{n} \tau \cdot \frac{1}{\tau}\left[2 \frac{\rho_{n}}{\alpha_{n}}\left(1-\alpha_{n} \tau\right)\left\langle\widetilde{A}_{1} x^{*}, x^{*}-T_{n} v_{n}\right\rangle\right. \\
\left.+\frac{\rho_{n}^{2}}{\alpha_{n}} \widetilde{M}_{3}^{2}+2 \mu\left\langle\widetilde{A}_{2} x^{*}, x^{*}-x_{n+1}\right\rangle\right] .
\end{aligned}
$$

Since $\sum_{n=0}^{\infty} \alpha_{n}=\infty, \rho_{n} \leq \alpha_{n}$ for all $n \geq 0$ and $\alpha_{n} \rightarrow 0$ as $n \rightarrow \infty$, we obtain, from (107) and (104) with $p=x^{*}$, that $\sum_{n=0}^{\infty} \alpha_{n} \tau=\infty, 2\left(\rho_{n} / \alpha_{n}\right)\left(1-\alpha_{n} \tau\right) \leq 2$, and

$$
\begin{aligned}
\limsup _{n \rightarrow \infty} \frac{1}{\tau}[ & {\left[2 \frac{\rho_{n}}{\alpha_{n}}\left(1-\alpha_{n} \tau\right)\left\langle\widetilde{A}_{1} x^{*}, x^{*}-T_{n} v_{n}\right\rangle\right.} \\
+ & \left.\frac{\rho_{n}^{2}}{\alpha_{n}} \widetilde{M}_{3}^{2}+2 \mu\left\langle\widetilde{A}_{2} x^{*}, x^{*}-x_{n+1}\right\rangle\right] \leq 0 .
\end{aligned}
$$

Applying Lemma 12 to (108), we infer that

$$
\lim _{n \rightarrow \infty}\left\|x_{n}-x^{*}\right\|=0 \text {. }
$$

This completes the proof.

In Theorem 18, putting $f(x) \equiv 0, \forall x \in C$, we obtain that $\Gamma=C$ and $T_{n}=I$ which is the identity mapping of $C$. Hence Theorem 18 reduces to the following.

Corollary 19. Let $C$ be a nonempty closed convex subset of a real Hilbert space $H$. Let $M, N, K \geq 1$ be three integers. Let $\Theta_{j}$ be a bifunction from $C \times C$ to $\mathbf{R}$ satisfying (A1)-(A4), $\varphi_{j}: C \rightarrow \mathbf{R}$ a lower semicontinuous and convex functional with the restriction (B1) or (B2), and $A_{j}: H \rightarrow H \zeta_{j}$-inverse strongly monotone for $j=1,2, \ldots, K$. Let $R_{k}: C \rightarrow 2^{H}$ be a maximal monotone mapping and let $B_{k}: C \rightarrow H$ be $\eta_{k}$-inverse strongly monotone for $k=1,2, \ldots, M$. Let $\left\{S_{i}\right\}_{i=1}^{N}$ be a finite family of nonexpansive mappings on $H$. Let $\widetilde{A}_{1}: H \rightarrow H$ be $\alpha$-inverse strongly monotone and let $\widetilde{A}_{2}:$ $H \rightarrow H$ be $\beta$-strongly monotone and $\kappa$-Lipschitz continuous. Assume that $\operatorname{VI}\left(\cap_{i=1}^{N} \operatorname{Fix}\left(S_{i}\right), \widetilde{A}_{1}\right) \neq \emptyset$ with $\left(\cap_{i=1}^{N} \operatorname{Fix}\left(S_{i}\right)\right) \quad \subset$ $\left(\cap_{j=1}^{K} \operatorname{GMEP}\left(\Theta_{j}, \varphi_{j}, A_{j}\right)\right) \cap\left(\cap_{k=1}^{M} I\left(B_{k}, R_{k}\right)\right)$. Let $\mu \in\left(0,2 \beta / \kappa^{2}\right)$, $\left\{\alpha_{n}\right\}_{n=0}^{\infty} \subset(0,1],\left\{\rho_{n}\right\}_{n=0}^{\infty} \subset(0,2 \alpha],\left\{\lambda_{k, n}\right\}_{n=0}^{\infty} \subset\left[a_{k}, b_{k}\right] \subset$ $\left(0,2 \eta_{k}\right)$, and $\left\{r_{j, n}\right\}_{n=0}^{\infty} \subset\left[c_{j}, d_{j}\right] \subset\left(0,2 \zeta_{j}\right)$ where $j \in$ $\{1,2, \ldots, K\}$ and $k \in\{1,2, \ldots, M\}$. For arbitrarily given $x_{0} \in$ $H$, let $\left\{x_{n}\right\}$ be a sequence generated by

$$
\begin{aligned}
u_{n}= & T_{r_{K, n}}^{\left(\Theta_{K}, \varphi_{K}\right)}\left(I-r_{K, n} A_{K}\right) T_{r_{K-1, n}}^{\left(\Theta_{K-1}, \varphi_{K-1}\right)}\left(I-r_{K-1, n} A_{K-1}\right) \\
& \cdots T_{r_{1, n}}^{\left(\Theta_{1}, \varphi_{1}\right)}\left(I-r_{1, n} A_{1}\right) x_{n}, \\
v_{n}= & J_{R_{M}, \lambda_{M, n}}\left(I-\lambda_{M, n} B_{M}\right) J_{R_{M-1}, \lambda_{M-1, n}}\left(I-\lambda_{M-1, n} B_{M-1}\right) \\
& \cdots J_{R_{1}, \lambda_{1, n}}\left(I-\lambda_{1, n} B_{1}\right) u_{n}, \\
y_{n}= & S_{[n+1]}\left(I-\rho_{n} \widetilde{A}_{1}\right) v_{n}, \\
x_{n+1}= & y_{n}-\mu \alpha_{n} \widetilde{A}_{2} y_{n}, \quad \forall n \geq 0 .
\end{aligned}
$$

Assume that

$$
\begin{aligned}
\bigcap_{i=1}^{N} \operatorname{Fix}\left(S_{i}\right) & =\operatorname{Fix}\left(S_{1} S_{2} \cdots S_{N}\right) \\
& =\operatorname{Fix}\left(S_{N} S_{1} \cdots S_{N-1}\right) \\
& =\cdots=\operatorname{Fix}\left(S_{2} S_{3} \cdots S_{N} S_{1}\right)
\end{aligned}
$$

and that the following conditions are satisfied:

(i) $\lim _{n \rightarrow \infty} \alpha_{n}=0, \sum_{n=0}^{\infty} \alpha_{n}=\infty$ and $\rho_{n} \leq \alpha_{n}$ for all $n \geq 0$

(ii) $\lim _{n \rightarrow \infty}\left(\left|\alpha_{n}-\alpha_{n+N}\right| /\left(\alpha_{n+N}\right)\right)=0$ or $\sum_{n=0}^{\infty}\left|\alpha_{n}-\alpha_{n+N}\right|<$ $\infty$;

(iii) $\lim _{n \rightarrow \infty}\left(\left|\rho_{n}-\rho_{n+N}\right| /\left(\rho_{n+N}\right)\right)=0$ or $\sum_{n=0}^{\infty}\left|\rho_{n}-\rho_{n+N}\right|<$ $\infty$;

(iv) $\lim _{n \rightarrow \infty}\left(\left|\lambda_{k, n}-\lambda_{k, n+N}\right| /\left(\alpha_{n}+N\right)\right)=0$ or $\sum_{n=0}^{\infty} \mid \lambda_{k, n}-$ $\lambda_{k, n+N} \mid<\infty$ for $k=1,2, \ldots, M$;

(v) $\lim _{n \rightarrow \infty}\left(\left|r_{j, n}-r_{j, n+N}\right| /\left(\alpha_{n}+N\right)\right)=0$ or $\sum_{n=0}^{\infty} \mid r_{j, n}-$ $r_{j, n+N} \mid<\infty$ for $j=1,2, \ldots, K$.

Then the following hold:

(a) $\left\{x_{n}\right\}_{n=0}^{\infty}$ is bounded;

(b) $\lim _{n \rightarrow \infty}\left\|x_{n}-x_{n+N}\right\|=0$;

(c) $\lim _{n \rightarrow \infty}\left\|x_{n}-S_{[n+N]} \cdots S_{[n+1]} x_{n}\right\|=0$ provided $\| x_{n}-$ $y_{n} \| \rightarrow 0(n \rightarrow \infty)$

(d) $\left\{x_{n}\right\}_{n=0}^{\infty}$ converges strongly to the unique element of $\operatorname{VI}\left(\operatorname{VI}\left(\cap_{i=1}^{N} \operatorname{Fix}\left(S_{i}\right), \widetilde{A}_{1}\right), \widetilde{A}_{2}\right)$ provided $\left\|x_{n}-y_{n}\right\|=$ $o\left(\rho_{n}\right)$.

In Corollary 19, putting $K=1$ and $M=2$, we obtain the following.

Corollary 20. Let $C$ be a nonempty closed convex subset of a real Hilbert space $H$. Let $N \geq 1$ be an integer. Let $\Theta$ be a bifunction from $C \times C$ to $\mathbf{R}$ satisfying (A1)-(A4), $\varphi$ : $C \rightarrow \mathbf{R}$ a lower semicontinuous and convex functional with the restriction (B1) or (B2), and $A: H \rightarrow H \zeta$-inverse strongly monotone. Let $R_{k}: C \rightarrow 2^{H}$ be a maximal monotone mapping and let $B_{k}: C \rightarrow H$ be $\eta_{k}$-inverse strongly monotone for $k=1,2$. Let $\left\{S_{i}\right\}_{i=1}^{N}$ be a finite family of nonexpansive mappings on $H$. Let $\widetilde{A}_{1}: H \rightarrow H$ be $\alpha$-inverse strongly monotone and let $\widetilde{A}_{2}: H \rightarrow H$ be $\beta$-strongly monotone and $\kappa$-Lipschitz continuous. Assume that $\operatorname{VI}\left(\cap_{i=1}^{N} \operatorname{Fix}\left(S_{i}\right), \widetilde{A}_{1}\right) \neq \emptyset$ with $\left(\cap_{i=1}^{N} \operatorname{Fix}\left(S_{i}\right)\right) \subset \operatorname{GMEP}(\Theta, \varphi, A) \cap I\left(B_{2}, R_{2}\right) \cap I\left(B_{1}, R_{1}\right)$. Let $\mu \in\left(0,2 \beta / \kappa^{2}\right),\left\{\alpha_{n}\right\}_{n=0}^{\infty} \subset(0,1],\left\{\rho_{n}\right\}_{n=0}^{\infty} \subset(0,2 \alpha]$, $\left\{\lambda_{k, n}\right\}_{n=0}^{\infty} \subset\left[a_{k}, b_{k}\right] \subset\left(0,2 \eta_{k}\right)$, and $\left\{r_{n}\right\}_{n=0}^{\infty} \subset[c, d] \subset(0,2 \zeta)$ 
for $k=1,2$. For arbitrarily given $x_{0} \in H$, let $\left\{x_{n}\right\}$ be a sequence generated by

$$
\begin{gathered}
\Theta\left(u_{n}, y\right)+\varphi(y)-\varphi\left(u_{n}\right)+\left\langle A x_{n}, y-u_{n}\right\rangle \\
+\frac{1}{r_{n}}\left\langle y-u_{n}, u_{n}-x_{n}\right\rangle \geq 0, \quad \forall y \in C, \\
v_{n}=J_{R_{2}, \lambda_{2, n}}\left(I-\lambda_{2, n} B_{2}\right) J_{R_{1}, \lambda_{1, n}}\left(I-\lambda_{1, n} B_{1}\right) u_{n}, \\
y_{n}=S_{[n+1]}\left(I-\rho_{n} \widetilde{A}_{1}\right) v_{n}, \\
x_{n+1}=y_{n}-\mu \alpha_{n} \widetilde{A}_{2} y_{n}, \quad \forall n \geq 0 .
\end{gathered}
$$

Assume that

$$
\begin{aligned}
\bigcap_{i=1}^{N} \operatorname{Fix}\left(S_{i}\right) & =\operatorname{Fix}\left(S_{1} S_{2} \cdots S_{N}\right) \\
& =\operatorname{Fix}\left(S_{N} S_{1} \cdots S_{N-1}\right) \\
& =\cdots=\operatorname{Fix}\left(S_{2} S_{3} \cdots S_{N} S_{1}\right)
\end{aligned}
$$

and that the following conditions are satisfied:

(i) $\lim _{n \rightarrow \infty} \alpha_{n}=0, \sum_{n=0}^{\infty} \alpha_{n}=\infty$ and $\rho_{n} \leq \alpha_{n}$ for all $n \geq 0$;

(ii) $\lim _{n \rightarrow \infty}\left(\left|\alpha_{n}-\alpha_{n+N}\right| /\left(\alpha_{n+N}\right)\right)=0$ or $\sum_{n=0}^{\infty}\left|\alpha_{n}-\alpha_{n+N}\right|<$ $\infty$;

(iii) $\lim _{n \rightarrow \infty}\left(\left|\rho_{n}-\rho_{n+N}\right| /\left(\rho_{n+N}\right)\right)=0$ or $\sum_{n=0}^{\infty}\left|\rho_{n}-\rho_{n+N}\right|<$ $\infty$;

(iv) $\lim _{n \rightarrow \infty}\left(\left|\lambda_{k, n}-\lambda_{k, n+N}\right| /\left(\alpha_{n+N}\right)\right)=0$ or $\sum_{n=0}^{\infty} \mid \lambda_{k, n}-$ $\lambda_{k, n+N} \mid<\infty$ for $k=1,2$;

(v) $\lim _{n \rightarrow \infty}\left(\left|r_{n}-r_{n+N}\right| /\left(\alpha_{n+N}\right)\right)=0$ or $\sum_{n=0}^{\infty}\left|r_{n}-r_{n+N}\right|<$ $\infty$.

Then the following hold:

(a) $\left\{x_{n}\right\}_{n=0}^{\infty}$ is bounded;

(b) $\lim _{n \rightarrow \infty}\left\|x_{n}-x_{n+N}\right\|=0$;

(c) $\lim _{n \rightarrow \infty}\left\|x_{n}-S_{[n+N]} \cdots S_{[n+1]} x_{n}\right\|=0$ provided $\| x_{n}-$ $y_{n} \| \rightarrow 0(n \rightarrow \infty)$;

(d) $\left\{x_{n}\right\}_{n=0}^{\infty}$ converges strongly to the unique element of $\operatorname{VI}\left(\operatorname{VI}\left(\cap_{i=1}^{N} \operatorname{Fix}\left(S_{i}\right), \widetilde{A}_{1}\right), \widetilde{A_{2}}\right)$ provided $\left\|x_{n}-y_{n}\right\|=$ $o\left(\rho_{n}\right)$.

In Theorem 18, putting $K=1$ and $M=2$, we obtain the following.

Corollary 21. Let $C$ be a nonempty closed convex subset of a real Hilbert space $H$ and let $f: C \rightarrow \mathbf{R}$ be a convex functional with L-Lipschitz continuous gradient $\nabla f$. Let $N \geq 1$ be an integer. Let $\Theta$ be a bifunction from $C \times C$ to $\mathbf{R}$ satisfying (A1)-(A4), $\varphi: C \rightarrow \mathbf{R}$ a lower semicontinuous and convex functional with the restriction (B1) or (B2), and $A: H \rightarrow H \zeta$ inverse-strongly monotone. Let $R_{k}: C \rightarrow 2^{H}$ be a maximal monotone mapping and let $B_{k}: C \rightarrow H$ be $\eta_{k}$-inverse strongly monotone for $k=1,2$. Let $\left\{S_{i}\right\}_{i=1}^{N}$ be a finite family of nonexpansive mappings on $H$. Let $\widetilde{A}_{1}: H \rightarrow H$ be $\alpha$-inverse strongly monotone and let $\widetilde{A}_{2}: H \rightarrow H$ be $\beta$ strongly monotone and $\kappa$-Lipschitz continuous. Assume that $\operatorname{VI}\left(\cap_{i=1}^{N} \operatorname{Fix}\left(S_{i}\right), \widetilde{A}_{1}\right) \neq \emptyset$ with $\cap_{i=1}^{N} \operatorname{Fix}\left(S_{i}\right) \subset \operatorname{GMEP}(\Theta, \varphi, A) \cap$ $I\left(B_{2}, R_{2}\right) \cap I\left(B_{1}, R_{1}\right) \cap \Gamma$. Let $\mu \in\left(0,2 \beta / \kappa^{2}\right),\left\{\alpha_{n}\right\}_{n=0}^{\infty} \subset(0,1]$, $\left\{\rho_{n}\right\}_{n=0}^{\infty} \subset(0,2 \alpha],\left\{\lambda_{k, n}\right\}_{n=0}^{\infty} \subset\left[a_{k}, b_{k}\right] \subset\left(0,2 \eta_{k}\right)$, and $\left\{r_{n}\right\}_{n=0}^{\infty} \subset$ $[c, d] \subset(0,2 \zeta)$ for $k=1,2$. For arbitrarily given $x_{0} \in H$, let $\left\{x_{n}\right\}$ be a sequence generated by

$$
\begin{gathered}
\Theta\left(u_{n}, y\right)+\varphi(y)-\varphi\left(u_{n}\right)+\left\langle A x_{n}, y-u_{n}\right\rangle \\
+\frac{1}{r_{n}}\left\langle y-u_{n}, u_{n}-x_{n}\right\rangle \geq 0, \quad \forall y \in C, \\
v_{n}=J_{R_{2}, \lambda_{2, n}}\left(I-\lambda_{2, n} B_{2}\right) J_{R_{1}, \lambda_{1, n}}\left(I-\lambda_{1, n} B_{1}\right) u_{n}, \\
y_{n}=S_{[n+1]}\left(I-\rho_{n} \widetilde{A}_{1}\right) T_{n} v_{n}, \\
x_{n+1}=y_{n}-\mu \alpha_{n} \widetilde{A}_{2} y_{n}, \quad \forall n \geq 0,
\end{gathered}
$$

where $P_{C}\left(I-\lambda_{n} \nabla f\right)=s_{n} I+\left(1-s_{n}\right) T_{n}$ (here $T_{n}$ is nonexpansive and $s_{n}:=s_{n}\left(\lambda_{n}\right)=\left(2-\lambda_{n} L\right) / 4 \in(0,1 / 2)$ for each $\lambda_{n} \in$ $(0,2 / L))$. Assume that

$$
\begin{aligned}
\bigcap_{i=1}^{N} \operatorname{Fix}\left(S_{i}\right) & =\operatorname{Fix}\left(S_{1} S_{2} \cdots S_{N}\right) \\
& =\operatorname{Fix}\left(S_{N} S_{1} \cdots S_{N-1}\right) \\
& =\cdots=\operatorname{Fix}\left(S_{2} S_{3} \cdots S_{N} S_{1}\right)
\end{aligned}
$$

and that the following conditions are satisfied:

(i) $\lim _{n \rightarrow \infty} \alpha_{n}=0, \sum_{n=0}^{\infty} \alpha_{n}=\infty$ and $\rho_{n} \leq \alpha_{n}$ for all $n \geq 0$;

(ii) $\lim _{n \rightarrow \infty}\left(\left|\alpha_{n}-\alpha_{n+N}\right| /\left(\alpha_{n+N}\right)\right)=0$ or $\sum_{n=0}^{\infty}\left|\alpha_{n}-\alpha_{n+N}\right|<$ $\infty$;

(iii) $\lim _{n \rightarrow \infty}\left(\left|s_{n}-s_{n+N}\right| /\left(\alpha_{n+N}\right)\right)=0$ or $\sum_{n=0}^{\infty}\left|s_{n}-s_{n+N}\right|<$ $\infty$;

(iv) $\lim _{n \rightarrow \infty}\left(\left|\rho_{n}-\rho_{n+N}\right| /\left(\rho_{n+N}\right)\right)=0$ or $\sum_{n=0}^{\infty}\left|\rho_{n}-\rho_{n+N}\right|<$ $\infty$;

(v) $\lim _{n \rightarrow \infty}\left(\left|\lambda_{k, n}-\lambda_{k, n+N}\right| /\left(\alpha_{n}+N\right)\right)=0$ or $\sum_{n=0}^{\infty} \mid \lambda_{k, n}-$ $\lambda_{k, n+N} \mid<\infty$ for $k=1,2$;

(vi) $\lim _{n \rightarrow \infty}\left(\left|r_{n}-r_{n+N}\right| /\left(\alpha_{n}+N\right)\right)=0$ or $\sum_{n=0}^{\infty}\left|r_{n}-r_{n+N}\right|<$ $\infty$.

Then the following hold:

(a) $\left\{x_{n}\right\}_{n=0}^{\infty}$ is bounded;

(b) $\lim _{n \rightarrow \infty}\left\|x_{n}-x_{n+N}\right\|=0$;

(c) $\lim _{n \rightarrow \infty}\left\|x_{n}-S_{[n+N]} \cdots S_{[n+1]} x_{n}\right\|=0$ provided $\lim _{n \rightarrow \infty}\left(\left\|x_{n}-y_{n}\right\|+\left\|T_{n} v_{n}-v_{n}\right\|\right)=0$

(d) $\left\{x_{n}\right\}_{n=0}^{\infty}$ converges strongly to the unique element of $\operatorname{VI}\left(\operatorname{VI}\left(\cap_{i=1}^{N} \operatorname{Fix}\left(S_{i}\right), \widetilde{A}_{1}\right), \widetilde{A}_{2}\right)$ provided $\left\|x_{n}-y_{n}\right\|+$ $\left\|T_{n} v_{n}-v_{n}\right\|=o\left(\rho_{n}\right)$.

In Theorem 18 , putting $K=1$ and $M=1$, we obtain the following. 
Corollary 22. Let $C$ be a nonempty closed convex subset of a real Hilbert space $H$ and let $f: C \rightarrow \mathbf{R}$ be a convex functional with L-Lipschitz continuous gradient $\nabla f$. Let $N \geq 1$ be an integer. Let $\Theta$ be a bifunction from $C \times C$ to $\mathbf{R}$ satisfying (A1)-(A4), $\varphi: C \rightarrow \mathbf{R}$ a lower semicontinuous and convex functional with the restriction (B1) or (B2), and $A: H \rightarrow H \zeta$ inverse-strongly monotone. Let $R: C \rightarrow 2^{H}$ be a maximal monotone mapping and let $B: C \rightarrow H$ be $\eta$-inverse strongly monotone. Let $\left\{S_{i}\right\}_{i=1}^{N}$ be a finite family of nonexpansive mappings on $H$. Let $\widetilde{A}_{1}: H \rightarrow H$ be $\alpha$-inverse strongly monotone and let $\widetilde{A}_{2}: H \rightarrow H$ be $\beta$-strongly monotone and $\kappa$-Lipschitz continuous. Assume that $\operatorname{VI}\left(\cap_{i=1}^{N} \operatorname{Fix}\left(S_{i}\right), \widetilde{A}_{1}\right) \neq \emptyset$ with $\left(\cap_{i=1}^{N} \operatorname{Fix}\left(S_{i}\right)\right) \subset \operatorname{GMEP}(\Theta, \varphi, A) \cap I(B, R) \cap \Gamma$. Let $\mu \in$ $\left(0,2 \beta / \kappa^{2}\right),\left\{\alpha_{n}\right\}_{n=0}^{\infty} \subset(0,1],\left\{\rho_{n}\right\}_{n=0}^{\infty} \subset(0,2 \alpha],\left\{\mu_{n}\right\}_{n=0}^{\infty} \subset$ $[a, b] \subset(0,2 \eta)$, and $\left\{r_{n}\right\}_{n=0}^{\infty} \subset[c, d] \subset(0,2 \zeta)$. For arbitrarily given $x_{0} \in H$, let $\left\{x_{n}\right\}$ be a sequence generated by

$$
\begin{gathered}
\Theta\left(u_{n}, y\right)+\varphi(y)-\varphi\left(u_{n}\right)+\left\langle A x_{n}, y-u_{n}\right\rangle \\
+\frac{1}{r_{n}}\left\langle y-u_{n}, u_{n}-x_{n}\right\rangle \geq 0, \quad \forall y \in C, \\
v_{n}=J_{R, \mu_{n}}\left(I-\mu_{n} B\right) u_{n}, \\
y_{n}=S_{[n+1]}\left(I-\rho_{n} \widetilde{A}_{1}\right) T_{n} v_{n}, \\
x_{n+1}=y_{n}-\mu \alpha_{n} \widetilde{A}_{2} y_{n}, \quad \forall n \geq 0,
\end{gathered}
$$

where $P_{C}\left(I-\lambda_{n} \nabla f\right)=s_{n} I+\left(1-s_{n}\right) T_{n}$ (here $T_{n}$ is nonexpansive and $s_{n}:=s_{n}\left(\lambda_{n}\right)=\left(2-\lambda_{n} L\right) / 4 \in(0,1 / 2)$ for each $\lambda_{n} \in$ $(0,2 / L))$. Assume that

$$
\begin{aligned}
\bigcap_{i=1}^{N} \operatorname{Fix}\left(S_{i}\right) & =\operatorname{Fix}\left(S_{1} S_{2} \cdots S_{N}\right) \\
& =\operatorname{Fix}\left(S_{N} S_{1} \cdots S_{N-1}\right) \\
& =\cdots=\operatorname{Fix}\left(S_{2} S_{3} \cdots S_{N} S_{1}\right)
\end{aligned}
$$

and that the following conditions are satisfied:

(i) $\lim _{n \rightarrow \infty} \alpha_{n}=0, \sum_{n=0}^{\infty} \alpha_{n}=\infty$ and $\rho_{n} \leq \alpha_{n}$ for all $n \geq 0$;

(ii) $\lim _{n \rightarrow \infty}\left(\left|\alpha_{n}-\alpha_{n+N}\right| /\left(\alpha_{n+N}\right)\right)=0$ or $\sum_{n=0}^{\infty}\left|\alpha_{n}-\alpha_{n+N}\right|<$ $\infty$;

(iii) $\lim _{n \rightarrow \infty}\left(\left|s_{n}-s_{n+N}\right| /\left(\alpha_{n+N}\right)\right)=0$ or $\sum_{n=0}^{\infty}\left|s_{n}-s_{n+N}\right|<$ $\infty$;

(iv) $\lim _{n \rightarrow \infty}\left(\left|\rho_{n}-\rho_{n+N}\right| /\left(\rho_{n+N}\right)\right)=0$ or $\sum_{n=0}^{\infty}\left|\rho_{n}-\rho_{n+N}\right|<$ $\infty$;

(v) $\lim _{n \rightarrow \infty}\left(\left|\mu_{n}-\mu_{n+N}\right| /\left(\alpha_{n}+N\right)\right)=0$ or $\sum_{n=0}^{\infty} \mid \mu_{n}-$ $\mu_{n+N} \mid<\infty$;

(vi) $\lim _{n \rightarrow \infty}\left(\left|r_{n}-r_{n+N}\right| /\left(\alpha_{n}+N\right)\right)=0$ or $\sum_{n=0}^{\infty}\left|r_{n}-r_{n+N}\right|<$ $\infty$.

Then the following hold:

(a) $\left\{x_{n}\right\}_{n=0}^{\infty}$ is bounded;

(b) $\lim _{n \rightarrow \infty}\left\|x_{n}-x_{n+N}\right\|=0$; (c) $\lim _{n \rightarrow \infty}\left\|x_{n}-S_{[n+N]} \cdots S_{[n+1]} x_{n}\right\|=0$ provided $\lim _{n \rightarrow \infty}\left(\left\|x_{n}-y_{n}\right\|+\left\|T_{n} v_{n}-v_{n}\right\|\right)=0 ;$

(d) $\left\{x_{n}\right\}_{n=0}^{\infty}$ converges strongly to the unique element of $\operatorname{VI}\left(\operatorname{VI}\left(\cap_{i=1}^{N} \operatorname{Fix}\left(S_{i}\right), \widetilde{A}_{1}\right), \widetilde{A_{2}}\right)$ provided $\left\|x_{n}-y_{n}\right\|+$ $\left\|T_{n} v_{n}-v_{n}\right\|=o\left(\rho_{n}\right)$.

\section{Conflict of Interests}

The authors declare that there is no conflict of interests regarding the publication of this paper.

\section{Acknowledgments}

This research was partially supported by the National Science Foundation of China (11071169), Innovation Program of Shanghai Municipal Education Commission (09ZZ133) and Ph.D. Program Foundation of Ministry of Education of China (20123127110002). This work was supported partly by the National Science Council of the Republic of China.

\section{References}

[1] J. Lions, Quelques Méthodes de Résolution des Problèmes aux Limites Non Linéaires, Dunod, Paris, France, 1969.

[2] G. M. Korpelevich, "The extragradient method for finding saddle points and other problems," Matecon, vol. 12, pp. 747756, 1976.

[3] L. C. Zeng and J. C. Yao, "Strong convergence theorem by an extragradient method for fixed point problems and variational inequality problems," Taiwanese Journal of Mathematics, vol. 10, no. 5, pp. 1293-1303, 2006.

[4] L. Ceng, Q. H. Ansari, and J. Yao, "Relaxed extragradient methods for finding minimum-norm solutions of the split feasibility problem," Nonlinear Analysis: Theory, Methods \& Applications, vol. 75, no. 4, pp. 2116-2125, 2012.

[5] L. C. Ceng, Q. H. Ansari, and J. C. Yao, "Relaxed extragradient iterative methods for variational inequalities," Applied Mathematics and Computation, vol. 218, no. 3, pp. 1112-1123, 2011.

[6] L. C. Ceng, Q. H. Ansari, N. C. Wong, and J. C. Yao, "An extragradient-like approximation method for variational inequalities and fixed point problems," Fixed Point Theory and Applications, vol. 2011, article 22, 18 pages, 2011.

[7] L. C. Ceng, M. Teboulle, and J. C. Yao, "Weak convergence of an iterative method for pseudomonotone variational inequalities and fixed-point problems," Journal of Optimization Theory and Applications, vol. 146, no. 1, pp. 19-31, 2010.

[8] J. Peng and J. Yao, "A new hybrid-extragradient method for generalized mixed equilibrium problems, fixed point problems and variational inequality problems," Taiwanese Journal of Mathematics, vol. 12, no. 6, pp. 1401-1432, 2008.

[9] Y. Yao, Y. C. Liou, and G. Marino, “Two-step iterative algorithms for hierarchical fixed point problems and variational inequality problems," Journal of Applied Mathematics and Computing, vol. 31, no. 1-2, pp. 433-445, 2009.

[10] L. Ceng and J. Yao, "A hybrid iterative scheme for mixed equilibrium problems and fixed point problems," Journal of Computational and Applied Mathematics, vol. 214, no. 1, pp. 186201, 2008. 
[11] C. Byrne, "A unified treatment of some iterative algorithms in signal processing and image reconstruction," Inverse Problems, vol. 20, no. 1, pp. 103-120, 2004.

[12] K. Goebel and W. A. Kirk, Topics on Metric Fixed-Point Theory, vol. 28 of Cambridge Studies in Advanced Mathematics, Cambridge University Press, 1990.

[13] H. K. Xu and T. H. Kim, "Convergence of hybrid steepestdescent methods for variational inequalities," Journal of Optimization Theory and Applications, vol. 119, no. 1, pp. 185-201, 2003.

[14] V. Barbu, Nonlinear Semigroups and Differential Equations in Banach Spaces, Noordhoff, 1976.

[15] N. Huang, "A new completely general class of variational inclusions with noncompact valued mappings," Computers \& Mathematics with Applications, vol. 35, no. 10, pp. 9-14, 1998.

[16] L. Ceng, Q. H. Ansari, M. M. Wong, and J. Yao, "Mann type hybrid extragradient method for variational inequalities, variational inclusions and fixed point problems," Fixed Point Theory, vol. 13, no. 2, pp. 403-422, 2012.

[17] L. C. Zeng, S. M. Guu, and J. C. Yao, "Characterization of $H$-monotone operators with applications to variational inclusions," Computers \& Mathematics with Applications, vol. 50, no. 3-4, pp. 329-337, 2005. 


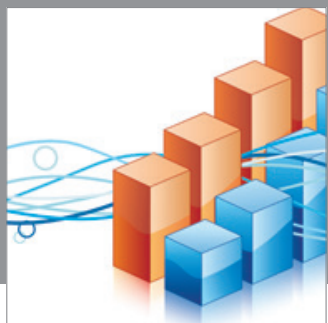

Advances in

Operations Research

mansans

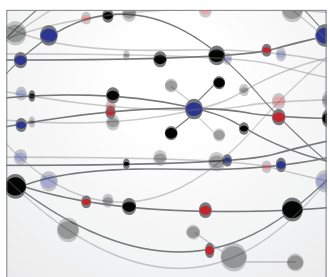

The Scientific World Journal
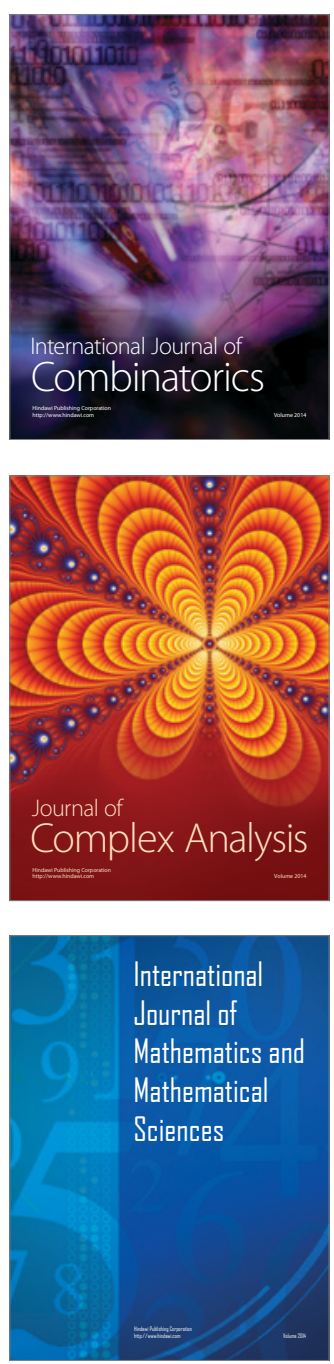
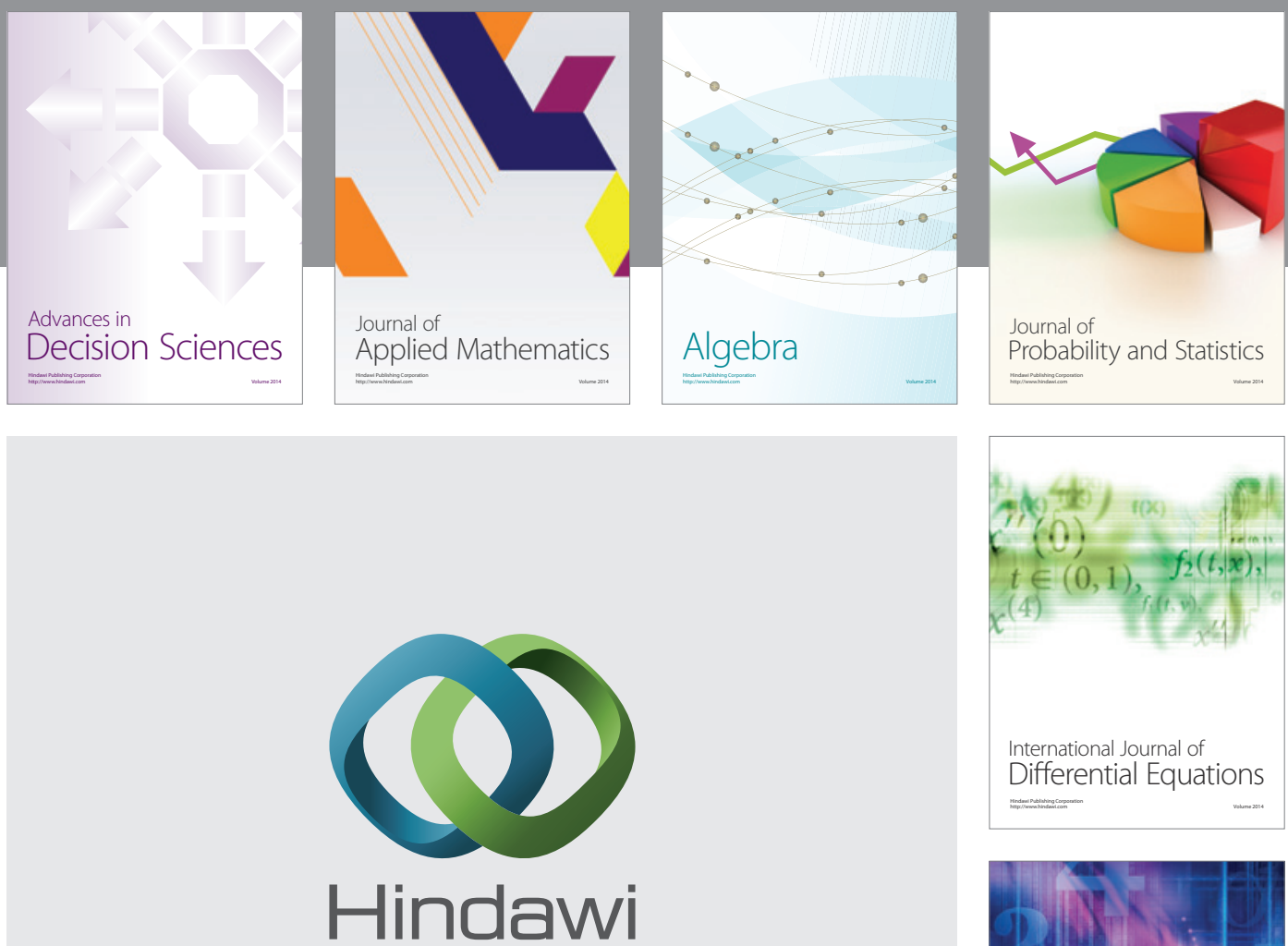

Submit your manuscripts at http://www.hindawi.com
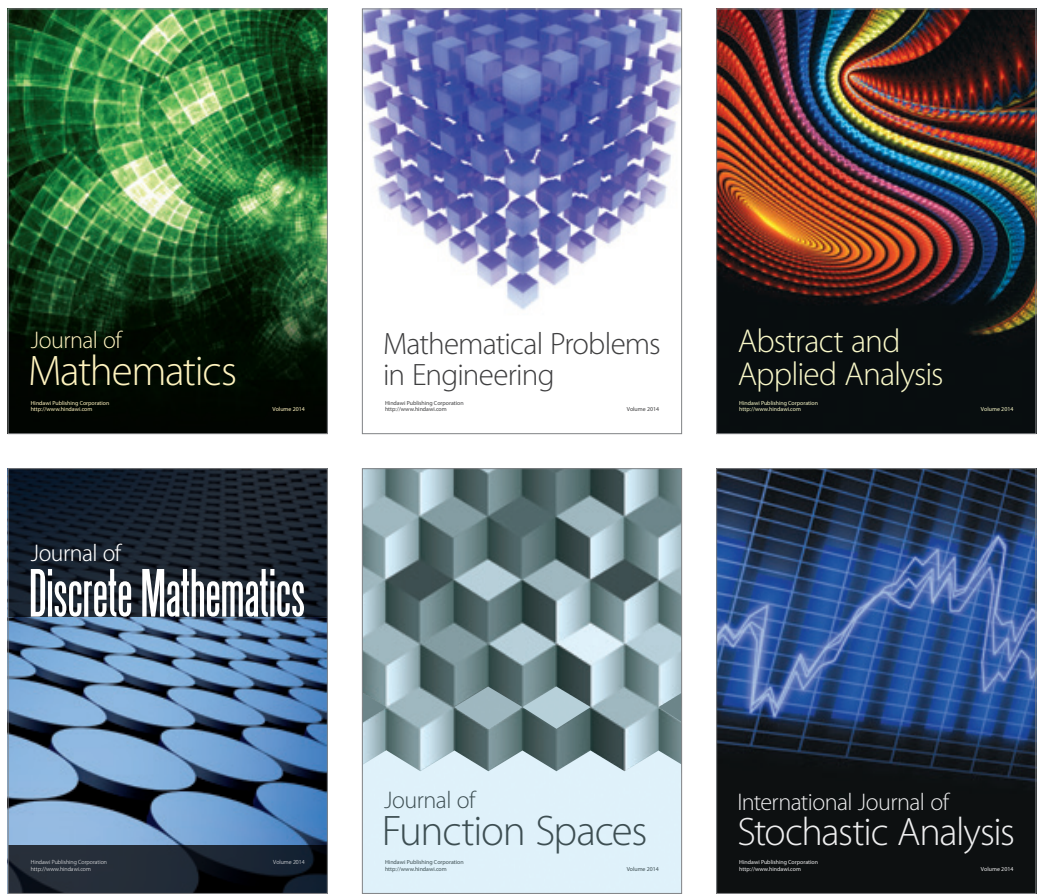

Journal of

Function Spaces

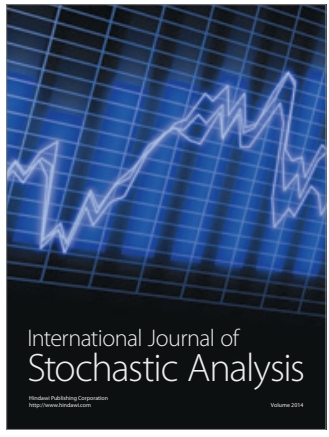

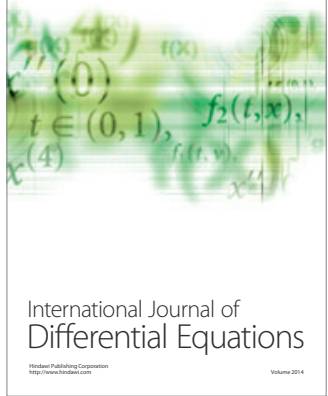
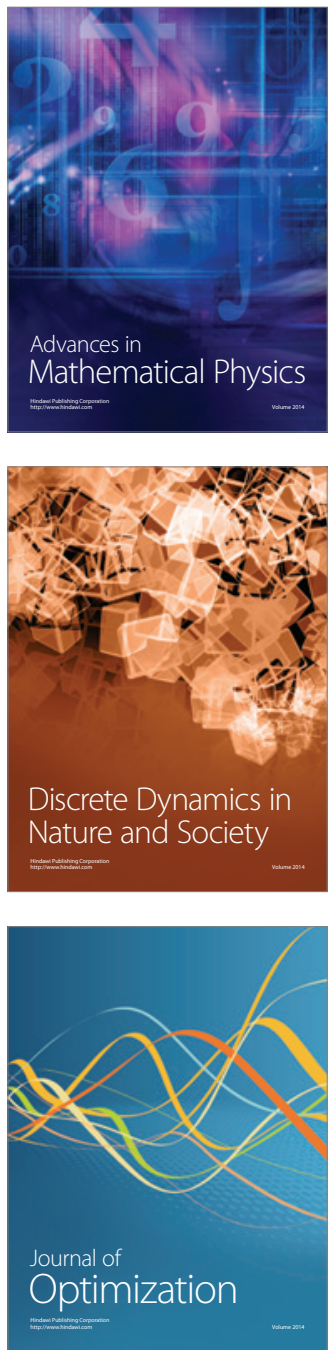